\title{
Jucys-Murphy elements, orthogonal matrix integrals, and Jack measures
}

\author{
SHo MATSUMOTO \\ Graduate School of Mathematics, Nagoya University. \\ Furocho, Chikusa-ku, Nagoya, 464-8602, JAPAN. \\ sho-matsumoto@math.nagoya-u.ac.jp
}

\begin{abstract}
We study symmetric polynomials whose variables are odd-numbered Jucys-Murphy elements. They define elements of the Hecke algebra associated to the Gelfand pair of the symmetric group with the hyperoctahedral group. We evaluate their expansions in zonal spherical functions and in double coset sums. These evaluations are related to integrals of polynomial functions over orthogonal groups. Furthermore, we give an extension of them, based on Jack polynomials.

2000 Mathematics Subject Classification: 05E15, 20C08, 05E05, 20C35.
\end{abstract}

\section{Introduction}

Let $S_{n}$ be the symmetric group. Jucys-Murphy elements are formal sums in the group algebra $\mathbb{C}\left[S_{n}\right]$ defined by

$$
J_{1}=0, \quad J_{k}=\sum_{i=1}^{k-1}(i k), \quad k=2,3, \ldots, n,
$$

where $(i k)$ is the transposition between $i$ and $k$. Every two of them commute with each other. Jucys [J] studied symmetric polynomials in variables $J_{1}, J_{2}, \ldots, J_{n}$ and proved that they are central elements in $\mathbb{C}\left[S_{n}\right]$. More precisely, given a symmetric function $F$, we have $F\left(J_{1}, J_{2}, \ldots, J_{n}\right) \in \mathcal{Z}_{n}$, where $\mathcal{Z}_{n}$ is the center of the group algebra $\mathbb{C}\left[S_{n}\right]$.

For a given element $F\left(J_{1}, J_{2}, \ldots, J_{n}\right) \in \mathcal{Z}_{n}$, it is a natural to ask for its character expansion and class expansion. Let $\chi^{\lambda}$ be the irreducible character of $S_{n}$ associated to Young diagram $\lambda$ and $f^{\lambda}$ the dimension of its corresponding representation. Jucys [J] obtained the character expansion

$$
F\left(J_{1}, J_{2}, \ldots, J_{n}\right)=\sum_{\lambda:|\lambda|=n} F\left(A_{\lambda}\right) \frac{f^{\lambda}}{n !} \chi^{\lambda}
$$

where $|\lambda|$ is the number of boxes in the Young diagram $\lambda$ and $A_{\lambda}$ is the alphabet of contents $j-i$ of boxes $(i, j)$ in $\lambda$.

The computation for the class expansion of $F\left(J_{1}, J_{2}, \ldots, J_{n}\right)$ is a more difficult problem. Let $\mathfrak{c}_{\mu}(n)$ be the sum of all permutations in $S_{n}$ of reduced cycle-type $\mu$. For example, $\mathfrak{c}_{(0)}(n)$ is the 
identity permutation and $\mathfrak{c}_{(1)}(n)$ is the sum of all transpositions. Then $\left\{\mathfrak{c}_{\mu}(n)|| \mu \mid+\ell(\mu) \leq n\right\}$ is a basis of $\mathcal{Z}_{n}$, where $\ell(\mu)$ is the number of rows of the diagram $\mu$. The question is to evaluate coefficients $\mathcal{A}_{\mu}(F, n)$ in

$$
F\left(J_{1}, J_{2}, \ldots, J_{n}\right)=\sum_{\mu:|\mu|+\ell(\mu) \leq n} \mathcal{A}_{\mu}(F, n) \mathfrak{c}_{\mu}(n)
$$

The $\mathcal{A}_{\mu}(F, n)$ are zero unless $\operatorname{deg} F \geq|\mu|$ and polynomials in $n$. If $\operatorname{deg} F=|\mu|$, then $\mathcal{A}_{\mu}(F, n)$ is independent of $n$. Lascoux and Thibon [LT] studied the coefficients for power-sum symmetric functions $F=p_{k}$, and Fujii et al. FKMO expressed $\mathcal{A}_{(0)}\left(p_{k}, n\right)$ as an explicit polynomial in binomail coefficients $\left(\begin{array}{l}n \\ m\end{array}\right)$. Matsumoto and Novak $[\mathrm{MN}$ gave a combinatorial explicit expression of $\mathcal{A}_{\mu}\left(m_{\lambda}, n\right)$ with $|\lambda|=|\mu|$, where $m_{\lambda}$ is the monomial symmetric function.

The coefficients $\mathcal{A}_{\mu}\left(h_{k}, n\right)$ for complete symmetric functions $h_{k}$ are more interesting. They appear in expansions of unitary matrix integrals. Let $U(N)$ be the group of $N \times N$ unitary matrices $g=\left(g_{i j}\right)_{1 \leq i, j \leq N}$, equipped with its normalized Haar measure $d g$. Consider integrals of the form

$$
\int_{g \in U(N)} g_{i_{1} j_{1}} g_{i_{2} j_{2}} \cdots g_{i_{n} j_{n}} \overline{g_{i_{1}^{\prime} j_{1}^{\prime}} g_{i_{2}^{\prime} j_{2}^{\prime}} \cdots g_{i_{n}^{\prime} j_{n}^{\prime}}} d g
$$

where $i_{k}, i_{k}^{\prime}, j_{k}, j_{k}^{\prime}$ are in $\{1,2, \ldots, N\}$ and $N \geq n$. The Weingarten calculus for unitary groups developed in [W, C, CS] states that those integrals are given by a sum of Weingarten functions

$$
\mathrm{Wg}_{n}^{U(N)}(\sigma)=\int_{g \in U(N)} \prod_{k=1}^{n} g_{k k} \overline{g_{k \sigma(k)}} d g, \quad \sigma \in S_{n} .
$$

In $[\mathbf{N}]$ (see also $[\mathrm{MN}]$ ), a remarkable connection between $\mathrm{Wg}_{n}^{U(N)}$ and Jucys-Murphy elements is discovered. Specifically, the Weingarten function is given as a generating function of $h_{k}\left(J_{1}, \ldots, J_{n}\right)$ :

$$
\sum_{\sigma \in S_{n}} \mathrm{Wg}_{n}^{U(N)}(\sigma) \sigma=\sum_{k=0}^{\infty}(-1)^{k} N^{-n-k} h_{k}\left(J_{1}, J_{2}, \ldots, J_{n}\right),
$$

or equivalently

$$
\mathrm{Wg}_{n}^{U(N)}(\sigma)=\sum_{k=0}^{\infty}(-1)^{k} N^{-n-k} \mathcal{A}_{\mu}\left(h_{k}, n\right),
$$

where $\mu$ is the reduced cycle-type of $\sigma$. Thus unitary matrix integrals are evaluated by observing symmetric functions in Jucys-Murphy elements.

The main purpose of the present paper is to study their analogues for orthogonal groups. In the orthogonal group case, the elements $F\left(J_{1}, J_{3}, \ldots, J_{2 n-1}\right) \cdot P_{n}$ are needed instead of $F\left(J_{1}, J_{2}, \ldots, J_{n}\right)$. Here $P_{n}=\sum_{\zeta \in H_{n}} \zeta$ is an element of $\mathbb{C}\left[S_{2 n}\right]$ and $H_{n}$ is the hyperoctahedral group realized in $S_{2 n}$. We will prove first that $F\left(J_{1}, J_{3}, \ldots, J_{2 n-1}\right) \cdot P_{n}$ belongs to the Hecke algebra $\mathcal{H}_{n}$ associated with the Gelfand pair $\left(S_{2 n}, H_{n}\right)$. The Hecke algebra $\mathcal{H}_{n}$ has two kinds of natural basis $\left\{\omega^{\lambda}|| \lambda \mid=n\right\}$ and $\left\{\psi_{\mu}(n)|| \mu \mid+\ell(\mu) \leq n\right\}$, where the $\omega^{\lambda}$ are zonal spherical functions and the $\psi_{\mu}(n)$ are sums over double cosets of the form $H_{n} \sigma H_{n}$. As in the unitary 
group case, it is natural to ask for expansions of $F\left(J_{1}, J_{3}, \ldots, J_{2 n-1}\right) \cdot P_{n}$ in zonal spherical functions $\omega^{\lambda}$ or in double coset sums $\psi_{\mu}(n)$. We will prove that the expansion in $\omega^{\lambda}$ is given by

$$
F\left(J_{1}, J_{3}, \ldots, J_{2 n-1}\right) \cdot P_{n}=\sum_{\lambda:|\lambda|=n} F\left(A_{\lambda}^{\prime}\right) \frac{f^{2 \lambda}}{(2 n-1) !} \omega^{\lambda},
$$

where $A_{\lambda}^{\prime}$ is the alphabet of modified contents $2 j-i-1$. Our main purpose is to obtain some properties for coefficients $\mathcal{A}_{\mu}^{\prime}(F, n)$ defined via

$$
F\left(J_{1}, J_{3}, \ldots, J_{2 n-1}\right) \cdot P_{n}=\sum_{\mu:|\mu|+\ell(\mu) \leq n} \mathcal{A}_{\mu}^{\prime}(F, n) \psi_{\mu}(n) .
$$

In general, the $\mathcal{A}_{\mu}^{\prime}(F, n)$ are different from $\mathcal{A}_{\mu}(F, n)$. For example, $\mathcal{A}_{(1)}\left(h_{3}, n\right)=\frac{1}{2} n^{2}+\frac{3}{2} n-4$ but $\mathcal{A}_{(1)}^{\prime}\left(h_{3}, n\right)=n^{2}+3 n-7$. However, by observing deep combinatorics of perfect matchings, we will prove that, if $\operatorname{deg} F=|\mu|$, they coincide as $\mathcal{A}_{\mu}(F, n)=\mathcal{A}_{\mu}^{\prime}(F, n)$, and are independent of $n$.

Like in the unitary group case, coefficients $\mathcal{A}_{\mu}^{\prime}\left(h_{k}, n\right)$ are involved in orthogonal matrix integrals. Let $O(N)$ be the orthogonal group of degree $N$ and $d g$ its normalized Haar measure. Then, for example, we obtain

$$
\int_{g \in O(N)} g_{11}^{2} g_{22}^{2} \cdots g_{n n}^{2} d g=\sum_{k=0}^{\infty}(-1)^{k} N^{-n-k} \mathcal{A}_{(0)}^{\prime}\left(h_{k}, n\right) .
$$

Via the Weingarten calculus for orthogonal groups developed in [CS, CM, Z], we establish the connection between orthogonal matrix integrals and Jucys-Murphy elements.

Furthermore, we introduce an $\alpha$-extension of $\mathcal{A}_{\mu}(F, n)$ and $\mathcal{A}_{\mu}^{\prime}(F, n)$. Let $\alpha$ be a positive real number. We define the value $\mathcal{A}_{\mu}^{(\alpha)}(F, n)$ as an average with respect to the Jack measure. The Jack measure is a probability measure on Young diagrams and is a deformation of the Plancherel measure for symmetric groups. Its definition is based on Jack polynomial theory and the connections between them and random matrix theory are much studied, see [Mat, BO] and their references. From symmetric function theory, we can see $\mathcal{A}_{\mu}(F, n)=\mathcal{A}_{\mu}^{(1)}(F, n)$ and $\mathcal{A}_{\mu}^{\prime}(F, n)=\mathcal{A}_{\mu}^{(2)}(F, n)$ for any symmetric function $F$ and partition $\mu$. Also $\mathcal{A}_{\mu}^{(1 / 2)}(F, n)$ are important and related to a twisted Gelfand pair.

We evaluate $\mathcal{A}_{\mu}^{(\alpha)}\left(e_{k}, n\right)$ for elementary symmetric functions $e_{k}$. Also, by applying shifted symmetric function theory developed in $\left[\mathrm{KOO}, \mathrm{L} 2, \mathrm{~L} 3, \mathrm{O}\right.$, we prove that the $\mathcal{A}_{\mu}^{(\alpha)}(F, n)$ are polynomials in $n$. We could not obtain any strong results for $\mathcal{A}_{\mu}^{(\alpha)}(F, n)$. Our appoarch is experimental but the author believes that it is fascinating and applicable in futurer research.

The present paper is constructed as follows: In Section 2 we review necessary notations and fundamental properties. In Section 3 , we evaluate $e_{k}\left(J_{1}, J_{3}, \ldots, J_{2 n-1}\right) \cdot P_{n}$ explicitly and prove that $F\left(J_{1}, J_{3}, \ldots, J_{2 n-1}\right) \cdot P_{n}$ is an element of the Hecke algebra $\mathcal{H}_{n}$. In Section 4 , we give the expansion of $F\left(J_{1}, J_{3}, \ldots, J_{2 n-1}\right) \cdot P_{n}$ in zonal spherical functions. In Section 5 an 6 , we give some properties of $\mathcal{A}_{\mu}^{\prime}(F, n)$. Specifically, we prove that $\mathcal{A}_{\mu}^{\prime}(F, n)$ coincides with $\mathcal{A}_{\mu}(F, n)$ if $|\mu|=\operatorname{deg} F$. As we mentioned, such an equality does not hold for $|\mu|<\operatorname{deg} F$. In Section 7, we see the connection to orthogonal matrix integrals. In Section 8, we study Jack's $\alpha$-deformations $\mathcal{A}_{\mu}^{(\alpha)}(F, n)$. In the final section, Section 9, we give some examples and suggest four conjectures. 
Remark 1.1. Since a primary version of this paper was released, all of our four conjectures given in Subsection 9.3 have been actively studied by some other reseachers. We would be able to see their proofs very soon.

\section{Preparations}

We use the notations of Macdonald. See Chapter I and VII in his book [Mac].

\subsection{Partitions and contents}

A partition $\lambda=\left(\lambda_{1}, \lambda_{2}, \ldots\right)$ is a weakly decreasing sequence of nonnegative integers such that its length $\ell(\lambda):=\left|\left\{i \geq 1 \mid \lambda_{i}>0\right\}\right|$ is finite. We write the size of $\lambda$ by $|\lambda|:=\sum_{i \geq 1} \lambda_{i}$. If $|\lambda|=n$, we say $\lambda$ to be a partition of $n$ and write $\lambda \vdash n$.

We often identify $\lambda$ with its Young diagram $Y(\lambda):=\left\{\square=(i, j) \in \mathbb{Z}^{2} \mid 1 \leq i \leq \ell(\lambda), 1 \leq\right.$ $j \leq \lambda_{i}$. If $\square=(i, j) \in Y(\lambda)$, we say that $\square$ is a box of $\lambda$ and write $\square \in \lambda$ shortly. The content of $\square=(i, j) \in \lambda$ is defined by $c(\square):=j-i$. Also we use its analogy $c^{\prime}(\square):=2 j-i-1$. Let $A_{\lambda}$ and $A_{\lambda}^{\prime}$ be the alphabet with $|\lambda|$ elements given by

$$
A_{\lambda}=\{c(\square) \mid \square \in \lambda\}, \quad A_{\lambda}^{\prime}=\left\{c^{\prime}(\square) \mid \square \in \lambda\right\} .
$$

For example, $A_{(2,2,1)}=\{1,0,0,-1,-2\}$ and $A_{(2,2,1)}^{\prime}=\{2,1,0,-1,-2\}$.

For each $i \geq 1$, we write the multiplicity of $i$ in $\lambda$ by $m_{i}(\lambda)=\left|\left\{j \geq 1 \mid \lambda_{j}=i\right\}\right|$. We sometimes write $\lambda$ as $\left(\ldots, 3^{m_{3}(\lambda)}, 2^{m_{2}(\lambda)}, 1^{m_{1}(\lambda)}\right)$. For example, $\lambda=(2,1,1,1)=\left(2,1^{3}\right)$. Define

$$
z_{\lambda}=\prod_{i \geq 1} i^{m_{i}(\lambda)} m_{i}(\lambda) !
$$

Let $\lambda, \mu$ be partitions. We define $\lambda+\mu$ to be the sequence of $\lambda_{i}+\mu_{i}:(\lambda+\mu)_{i}=\lambda_{i}+\mu_{i}$. Also we define $\lambda \cup \mu$ to be the partition whose parts are those of $\lambda$ and $\mu$, arranged in decreasing order. In general, given partitions $\lambda^{(1)}, \lambda^{(2)}, \ldots, \lambda^{(k)}$, we define $\lambda^{(1)} \cup \lambda^{(2)} \cup \cdots \cup \lambda^{(k)}$ in the same way.

For a partition $\lambda$ with $\ell(\lambda)=l$, we define its reduction $\tilde{\lambda}$ by $\tilde{\lambda}=\left(\lambda_{1}-1, \lambda_{2}-1, \ldots, \lambda_{l}-1\right)$. For each $n \geq 1$, the map $\lambda \mapsto \tilde{\lambda}$ gives a bijection from the set $\{\lambda|| \lambda \mid=n\}$ to $\{\mu|| \mu \mid+\ell(\mu) \leq n\}$. Indeed, its inverse map is given by $\mu \mapsto \mu+\left(1^{n-|\mu|}\right)=: \lambda$. Then $|\lambda|-\ell(\lambda)=|\mu|$.

\subsection{Symmetric functions}

Let $x=\left(x_{1}, x_{2}, \ldots\right)$ be an infinite sequence of indeterminates, and $\mathbb{S}$ the algebra of symmetric functions with complex coefficients in variables $x$.

Given a partition $\lambda$, the monomial symmetric function $m_{\lambda}$ is defined by

$$
m_{\lambda}(x)=\sum_{\left(\alpha_{1}, \alpha_{2}, \ldots\right)} x_{1}^{\alpha_{1}} x_{2}^{\alpha_{2}} \cdots
$$


summed over all distinct permutations $\left(\alpha_{1}, \alpha_{2}, \ldots\right)$ of $\left(\lambda_{1}, \lambda_{2}, \ldots\right)$. Denote by $e_{k}, p_{k}$, and $h_{k}$ the elementary, power-sum, and complete symmetric functions, respectively. Namely,

$$
\begin{aligned}
& e_{k}(x)=m_{\left(1^{k}\right)}(x)=\sum_{i_{1}<i_{2}<\cdots<i_{k}} x_{i_{1}} x_{i_{2}} \cdots x_{i_{k}}, \\
& p_{k}(x)=m_{(k)}(x)=x_{1}^{k}+x_{2}^{k}+\cdots, \\
& h_{k}(x)=\sum_{\lambda \vdash k} m_{\lambda}(x)=\sum_{i_{1} \leq i_{2} \leq \cdots \leq i_{k}} x_{i_{1}} x_{i_{2}} \cdots x_{i_{k}} .
\end{aligned}
$$

Also we put $e_{\lambda}=\prod_{i=1}^{\ell(\lambda)} e_{\lambda_{i}}$, and similarly for $p_{\lambda}$ and $h_{\lambda}$. For convenience, we set $m_{(0)}=e_{0}=$ $h_{0}=1$.

For finite variables $\left(x_{1}, x_{2}, \ldots, x_{n}\right)$, the monomial symmetric function (or polynomial) $m_{\lambda}\left(x_{1}, x_{2}, \ldots\right)$ is zero unless $\ell(\lambda) \leq n$.

The degree of $m_{\lambda}$ is naturally defined to be $\operatorname{deg} m_{\lambda}=|\lambda|$.

The fundamental theorem for symmetric functions says that any symmetric function $F$ is given by a polynomial in $e_{1}, e_{2}, \ldots$ and that the $e_{k}$ are algebraically independent. We can replace $e_{k}$ by $p_{k}$ in this statement.

\subsection{Hyperoctahedral groups}

We recall a Gelfand pair $\left(S_{2 n}, H_{n}\right)$. The detail is seen in [Mac, VII.2].

Let $S_{n}$ be the symmetric group on $\{1,2, \ldots, n\}$. Let $\mathbb{C}\left[S_{n}\right]$ denote the algebra of all complexvalued functions $f$ on $S_{n}$ under convolution $\left(f_{1} \cdot f_{2}\right)(\sigma)=\sum_{\tau \in S_{n}} f_{1}\left(\sigma \tau^{-1}\right) f_{2}(\tau)$. This is identified with the algebra of formal $\mathbb{C}$-linear sums of permutations with multiplication $\left(\sum_{\sigma} f_{1}(\sigma) \sigma\right)\left(\sum_{\tau} f_{2}(\tau) \tau\right)=$ $\sum_{\pi}\left(\sum_{\sigma} f_{1}(\sigma) f_{2}\left(\sigma^{-1} \pi\right)\right) \pi$.

A permutation $\sigma$ in $S_{n}$ is regarded as a permutation in $S_{n+1}$ fixing the letter $n+1$. Thus $\mathbb{C}\left[S_{n}\right] \subset \mathbb{C}\left[S_{n+1}\right]$.

Let $H_{n}$ be the hyperoctahedral group, which is a subgroup of $S_{2 n}$ generated by transpositions $(2 i-12 i),(1 \leq i \leq n)$, and by double transpositions $(2 i-12 j-1)(2 i 2 j),(1 \leq i<j \leq n)$. Equivalently, $H_{n}$ is the centralizer of $\left(\begin{array}{l}1 \\ 2\end{array}\right)(34) \cdots(2 n-12 n)$ in $S_{2 n}$. Then the pair $\left(S_{2 n}, H_{n}\right)$ is a Gelfand pair. Let $P_{n}$ be the sum of all elements of $H_{n}$ in $\mathbb{C}\left[S_{2 n}\right]$ :

$$
P_{n}=\sum_{\zeta \in H_{n}} \zeta
$$

Consider the double cosets $H_{n} \sigma H_{n}$ in $S_{2 n}$. These cosets are indexed by partitions of $n$, that is,

$$
S_{2 n}=\bigsqcup_{\rho \vdash n} H_{\rho},
$$

where each $H_{\rho}$ is a double coset. The permutation $\sigma \in S_{2 n}$ is said to be of coset-type $\rho$ and written as $\Xi_{n}(\sigma)=\rho$ if $\sigma \in H_{\rho}$.

Also the partition $\Xi_{n}(\sigma)$ is graphically defined as follows. Consider the graph $\Gamma(\sigma)$ whose vertex set is $\{1,2, \ldots, 2 n\}$ and whose edge set consists of $\{2 i-1,2 i\}$ and $\{\sigma(2 i-1), \sigma(2 i)\}$, 
$1 \leq i \leq n$. We think of the edges $\{\sigma(2 i-1), \sigma(2 i)\}$ as blue, and the others as red. Then $\Gamma(\sigma)$ has some connected components of even lengths $2 \rho_{1} \geq 2 \rho_{2} \geq \cdots$. Thus $\sigma$ determines a partition $\rho=\left(\rho_{1}, \rho_{2}, \ldots\right)$ of $n$. The $\rho$ is nothing but the coset-type $\Xi_{n}(\sigma)$.

Two permutations $\sigma_{1}, \sigma_{2} \in S_{2 n}$ have the same coset-type if and only if $H_{n} \sigma_{1} H_{n}=H_{n} \sigma_{2} H_{n}$. The set $H_{\rho}$ consists of permutations in $S_{2 n}$ of coset-type $\rho$. Given $\sigma \in S_{2 n}$, we let $\nu_{n}(\sigma)$ to be the length of the partition $\Xi_{n}(\sigma): \nu_{n}(\sigma)=\ell\left(\Xi_{n}(\sigma)\right)$.

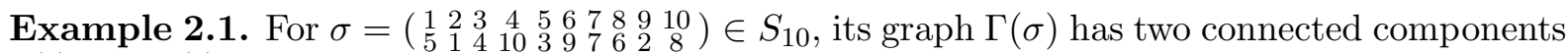
$\Gamma^{(1)}$ and $\Gamma^{(2)}$ :

$$
\Gamma^{(1)}: 1 \Longleftrightarrow 5 \longleftrightarrow 6 \Longleftrightarrow 7 \longleftrightarrow 8 \Longleftrightarrow 2 \longleftrightarrow 1, \quad \Gamma^{(2)}: 3 \Longleftrightarrow 9 \longleftrightarrow 10 \Longleftrightarrow 4 \longleftrightarrow 3 .
$$

Here " $i \Longleftrightarrow j$ " means that a blue edge connects the $i$-th vertex with the $j$-th vertex, whereas " $p \longleftrightarrow q$ " means that a red edge connects the $p$-th vertex with the $q$-th vertex. Equivalently, there exists an integer $k$ such that $\{i, j\}=\{\sigma(2 k-1), \sigma(2 k)\}$ (resp. $\{p, q\}=\{2 k-1,2 k\}$ ). In this example, the component $\Gamma^{(1)}$ and $\Gamma^{(2)}$ has 6 and 4 vertices, respectively, and hence $\Xi_{5}(\sigma)=(3,2)$ and $\nu_{5}(\sigma)=2$.

\subsection{Perfect matchings}

Let $\mathcal{M}(2 n)$ be the set of all perfect matchings on $\{1,2, \ldots, 2 n\}$. Each perfect matching $\mathfrak{m}$ in $\mathcal{M}(2 n)$ is uniquely expressed by the form

$$
\{\{\mathfrak{m}(1), \mathfrak{m}(2)\},\{\mathfrak{m}(3), \mathfrak{m}(4)\}, \ldots,\{\mathfrak{m}(2 n-1), \mathfrak{m}(2 n)\}\}
$$

with $\mathfrak{m}(2 i-1)<\mathfrak{m}(2 i)$ for $1 \leq i \leq n$ and with $\mathfrak{m}(1)<\mathfrak{m}(3)<\cdots<\mathfrak{m}(2 n-1)$. We call each $\{\mathfrak{m}(2 i-1), \mathfrak{m}(2 i)\}$ a block of $\mathfrak{m}$. A block of the form $\{2 i-1,2 i\}$ is said to be trivial. We embed the set $\mathcal{M}(2 n)$ into $S_{2 n}$ via the mapping

$$
\mathcal{M}(2 n) \ni \mathfrak{m} \mapsto\left(\begin{array}{cccccc}
1 & 2 & 3 & 4 & \cdots & 2 n \\
\mathfrak{m}(1) & \mathfrak{m}(2) & \mathfrak{m}(3) & \mathfrak{m}(4) & \cdots & \mathfrak{m}(2 n)
\end{array}\right) \in S_{2 n}
$$

In particular, the graph $\Gamma(\mathfrak{m})$, the coset-type $\Xi_{n}(\mathfrak{m})$, and the value $\nu_{n}(\mathfrak{m})$ are defined. Note that $\Gamma(\mathfrak{m})=\Gamma(\mathfrak{n})$ if and only if $\mathfrak{m}=\mathfrak{n}$.

A perfect matching $\mathfrak{n}$ in $\mathcal{M}(2 n-2)$ is regarded as an element of $\mathcal{M}(2 n)$ by adding the trivial block $\{2 n-1,2 n\}$ :

$$
\mathcal{M}(2 n-2) \ni \mathfrak{n} \mapsto \mathfrak{n} \sqcup\{\{2 n-1,2 n\}\} \in \mathcal{M}(2 n) .
$$

Thus we think as $\mathcal{M}(2 n-2) \subset \mathcal{M}(2 n)$.

It is well known that $\mathcal{M}(2 n)$ is the set of all representatives of the right cosets $\sigma H_{n}$ of $H_{n}$ in $S_{2 n}$ :

$$
S_{2 n}=\bigsqcup_{\mathfrak{m} \in \mathcal{M}(2 n)} \mathfrak{m} H_{n}
$$




\subsection{Characters and zonal spherical functions}

Given a partition $\lambda \vdash n$, we denote by $\chi^{\lambda}$ the irreducible character of $S_{n}$. The set $\left\{\chi^{\lambda} \mid \lambda \vdash n\right\}$ is a basis of the center of the group algebra $\mathbb{C}\left[S_{n}\right]$. Let $\operatorname{id}_{n}$ denote the identity permutation in $S_{n}$ and let $f^{\lambda}:=\chi^{\lambda}\left(\operatorname{id}_{n}\right)$. Thus the number $f^{\lambda}$ is the dimension of the irreducible representation of $S_{n}$ with character $\chi^{\lambda}$. Equivalently, $f^{\lambda}$ is the number of standard Young tableaux of shape $\lambda$, see e.g. [Sa]. by

For each partition $\lambda$ of $n$, we define the zonal spherical function of the Gelfand pair $\left(S_{2 n}, H_{n}\right)$

$$
\omega^{\lambda}(\sigma)=\frac{1}{2^{n} n !} \sum_{\zeta \in H_{n}} \chi^{2 \lambda}(\sigma \zeta), \quad \sigma \in S_{2 n},
$$

where $2 \lambda=\lambda+\lambda=\left(2 \lambda_{1}, 2 \lambda_{2}, \ldots\right)$. If we regard $\omega^{\lambda}$ as an element of $\mathbb{C}\left[S_{2 n}\right]$, we can express $\omega^{\lambda}=\frac{1}{2^{n} n !} \chi^{2 \lambda} P_{n}=\frac{1}{2^{n} n !} P_{n} \chi^{2 \lambda}$. These functions are $H_{n}$-biinvariant functions on $S_{2 n}$ and constant on each double coset $H_{\rho}$. Denote by $\omega_{\rho}^{\lambda}$ the value of $\omega^{\lambda}$ at $H_{\rho}$.

Let $\mathcal{H}_{n}$ be the Hecke algebra associated with the Gelfand pair $\left(S_{2 n}, H_{n}\right)$ :

$$
\mathcal{H}_{n}=\left\{f: S_{2 n} \rightarrow \mathbb{C} \mid f \text { is constant on each } H_{\rho}(\rho \vdash n)\right\} .
$$

Since $\left(S_{2 n}, H_{n}\right)$ is a Gelfand pair, this algebra is commutative with respect to the convolution product. We often regard $\mathcal{H}_{n}$ as a subspace of $\mathbb{C}\left[S_{2 n}\right]$. There are two natural bases of $\mathcal{H}_{n}$; one is $\left\{\omega^{\lambda} \mid \lambda \vdash n\right\}$ and another is $\left\{\phi_{\rho} \mid \rho \vdash n\right\}$. Here the $\phi_{\rho}$ are double-coset sum functions

$$
\phi_{\rho}=\sum_{\sigma \in H_{\rho}} \sigma .
$$

Note that $\phi_{\left(1^{n}\right)}=P_{n}$.

\section{Analogue of Jucys' result}

Define the Jucys-Murphy elements $J_{k}$. They are commuting elements in group algebras of symmetric groups, given by $J_{1}=0$ and by

$$
J_{k}=(1 k)+(2 k)+\cdots+(k-1 k) \quad \text { for } k=2,3, \ldots
$$

Jucys [J] obtains an exact expression for $e_{k}\left(J_{1}, J_{2}, \ldots, J_{n}\right)$, where $e_{k}$ is the elementary symmetric function. His result is the following identity:

$$
e_{k}\left(J_{1}, J_{2}, \ldots, J_{n}\right)=\sum_{\pi} \pi
$$

summed over all permutations $\pi$ in $S_{n}$ with exactly $n-k$ cycles (including trivial cycles). The following proposition is an analogue of this identity, and was essentially obtained by Zinn-Justin [Z] very recently. Our proof is an analogue of Jucys' proof in [J]. 
Proposition 3.1. For any $k$ and $n$, we have

$$
e_{k}\left(J_{1}, J_{3}, \ldots, J_{2 n-1}\right) \cdot P_{n}=\sum_{\substack{\mathfrak{m} \in \mathcal{M}(2 n) \\ \nu_{n}(\mathfrak{m})=n-k}} \mathfrak{m} P_{n} .
$$

Proof. First observe that (3.1) holds true when $k=0$ because $\mathfrak{m}=\{\{1,2\},\{3,4\}, \ldots,\{2 n-$ $1,2 n\}\}$ is the unique element in $\mathcal{M}(2 n)$ satisfying $\nu_{n}(\mathfrak{m})=n$. Also, when $k \geq n$, both sides are zero.

We proceed by induction on $n$. Let $n>1$ and suppose that the claim holds for $e_{k}\left(J_{1}, J_{3}, \ldots, J_{2 n-3}\right)$. $P_{n-1}$ with any $k \geq 0$. Using identities $e_{k}\left(x_{1}, x_{2}, \ldots, x_{n}\right)=e_{k}\left(x_{1}, \ldots, x_{n-1}\right)+x_{n} e_{k-1}\left(x_{1}, \ldots, x_{n-1}\right)$ and $P_{n-1} P_{n}=\left|H_{n-1}\right| P_{n}$, we see that

$$
\begin{aligned}
& e_{k}\left(J_{1}, J_{3}, \ldots, J_{2 n-1}\right) P_{n} \\
= & e_{k}\left(J_{1}, J_{3}, \ldots, J_{2 n-3}\right) P_{n}+J_{2 n-1} e_{k-1}\left(J_{1}, J_{3}, \ldots, J_{2 n-3}\right) P_{n} \\
= & \frac{1}{\left|H_{n-1}\right|} e_{k}\left(J_{1}, J_{3}, \ldots, J_{2 n-3}\right) P_{n-1} P_{n}+\frac{1}{\left|H_{n-1}\right|} J_{2 n-1} e_{k-1}\left(J_{1}, J_{3}, \ldots, J_{2 n-3}\right) P_{n-1} P_{n} .
\end{aligned}
$$

The induction assumption gives

$$
e_{k}\left(J_{1}, J_{3}, \ldots, J_{2 n-1}\right) P_{n}=\sum_{\substack{\mathfrak{n} \in \mathcal{M}(2 n-2) \\ \nu_{n-1}(\mathfrak{n})=n-1-k}} \mathfrak{n} P_{n}+\sum_{t=1}^{2 n-2} \sum_{\substack{\mathfrak{n} \in \mathcal{M}(2 n-2) \\ \nu_{n-1}(\mathfrak{n})=n-k}}(t 2 n-1) \mathfrak{n} P_{n} .
$$

Recall the natural inclusion $\mathcal{M}(2 n-2) \ni \mathfrak{n} \mapsto \mathfrak{n} \sqcup\{\{2 n-1,2 n\}\} \in \mathcal{M}(2 n)$. We have $\nu_{n}(\mathfrak{n} \sqcup\{\{2 n-1,2 n\}\})=\nu_{n-1}(\mathfrak{n})+1$, and hence the first summand on the right hand side of (3.2) coincides with $\sum_{\mathfrak{m}} \mathfrak{m} P_{n}$, summed over $\mathfrak{m} \in \mathcal{M}(2 n)$ having the block $\{2 n-1,2 n\}$ with $\nu_{n}(\mathfrak{m})=n-k$.

Next, let us see $(t 2 n-1) \mathfrak{n} P_{n}$ for $\mathfrak{n} \in \mathcal{M}(2 n-2)$ and $1 \leq t \leq 2 n-2$. Denote by $t_{\mathfrak{n}}$ the index in $\{1,2, \ldots, 2 n-2\}$, determined by $\left\{t_{\mathfrak{n}}, t\right\} \in \mathfrak{n}$. We define an element $\mathfrak{n}_{t}$ in $\mathcal{M}(2 n)$ by removing $\left\{t_{\mathfrak{n}}, t\right\}$ from $\mathfrak{n}$ and by adding two new blocks $\left\{t_{\mathfrak{n}}, 2 n-1\right\}$ and $\{t, 2 n\}$ :

$$
\mathfrak{n}_{t}=\left(\mathfrak{n} \backslash\left\{\left\{t_{\mathfrak{n}}, t\right\}\right\}\right) \cup\left\{\left\{t_{\mathfrak{n}}, 2 n-1\right\},\{t, 2 n\}\right\} .
$$

Then it is easy to see $\mathfrak{n}_{t} H_{n}=(t 2 n-1) \mathfrak{n} H_{n}$, and therefore

$$
\mathfrak{n}_{t} P_{n}=(t 2 n-1) \mathfrak{n} P_{n}
$$

Moreover, we can see

$$
\nu_{n-1}(\mathfrak{n})=\nu_{n}\left(\mathfrak{n}_{t}\right) .
$$

In fact, consider graphs $\Gamma(\mathfrak{n}), \Gamma\left(\mathfrak{n}_{t}\right)$ defined in Subsection 2.3. We use the notation of Example 2.1. The graph $\Gamma\left(\mathfrak{n}_{t}\right)$ can be obtained from $\Gamma(\mathfrak{n})$ if we replace an edge $t_{\mathfrak{n}} \Longleftrightarrow t$ in $\Gamma(\mathfrak{n})$ by the path $t_{\mathfrak{n}} \Longleftrightarrow 2 n-1 \longleftrightarrow 2 n \Longleftrightarrow t$. This means that the numbers of components of $\Gamma(\mathfrak{n})$ and $\Gamma\left(\mathfrak{n}_{t}\right)$ coincide, i.e., $\nu_{n-1}(\mathfrak{n})=\nu_{n}\left(\mathfrak{n}_{t}\right)$.

The observation in the previous paragraph implies that for each $t$ the $\operatorname{sum} \sum_{\mathfrak{n}}(t 2 n-1) \mathfrak{n} P_{n}$ on (3.2) coincides with $\sum_{\mathfrak{m}} \mathfrak{m} P_{n}$, summed over $\mathfrak{m} \in \mathcal{M}(2 n)$ having the block $\{t, 2 n\}$ with $\nu_{n}(\mathfrak{m})=$ $n-k$.

It follows that the expression (3.2) is $\sum_{\mathfrak{m}} \mathfrak{m} P_{n}$, summed over all $\mathfrak{m} \in \mathcal{M}(2 n)$ with $\nu_{n}(\mathfrak{m})=$ $n-k$. Thus (3.1) is proved. 
Recall that $H_{\rho}$ is the double coset $H_{n} \sigma H_{n}$ of permutations of coset-type $\rho$, and that $\phi_{\rho}$ is the formal sum over $H_{\rho}$ in $\mathbb{C}\left[S_{2 n}\right]$.

Corollary 3.2. For each $0 \leq k<n$, we have

$$
e_{k}\left(J_{1}, J_{3}, \ldots, J_{2 n-1}\right) \cdot P_{n}=\sum_{\substack{\rho \vdash n \\ \ell(\rho)=n-k}} \phi_{\rho} .
$$

This belongs to $\mathcal{H}_{n}$. Moreover, $e_{k}\left(J_{1}, J_{3}, \ldots, J_{2 n-1}\right) \cdot P_{n}=P_{n} \cdot e_{k}\left(J_{1}, J_{3}, \ldots, J_{2 n-1}\right)$.

Proof. By decompositions (2.5) and (2.2), the right hand side on (3.1) equals

$$
\sum_{\substack{\mathfrak{m} \in \mathcal{M}(2 n) \\ \nu_{n}(\mathfrak{m})=n-k}} \sum_{\zeta \in H_{n}} \mathfrak{m} \zeta=\sum_{\substack{\sigma \in S_{2 n} \\ \nu_{n}(\sigma)=n-k}} \sigma=\sum_{\substack{\rho \vdash n \\ \ell(\rho)=n-k}} \sum_{\sigma \in H_{\rho}} \sigma,
$$

which implies (3.3). Let $\iota: \mathbb{C}\left[S_{2 n}\right] \rightarrow \mathbb{C}\left[S_{2 n}\right]$ be the linear extension of the anti-isomorphism $S_{2 n} \ni \sigma \mapsto \sigma^{-1} \in S_{2 n}$. By (3.3) and by the fact that $H_{n} \sigma H_{n}=H_{n} \sigma^{-1} H_{n}$ for any $\sigma \in S_{2 n}$, the element $e_{k}\left(J_{1}, J_{3}, \ldots, J_{2 n-1}\right) \cdot P_{n}$ is invariant under $\iota$. However, $\iota\left(e_{k}\left(J_{1}, J_{3}, \ldots, J_{2 n-1}\right) \cdot P_{n}\right)=$ $\iota\left(P_{n}\right) \cdot \iota\left(e_{k}\left(J_{1}, J_{3}, \ldots, J_{2 n-1}\right)\right)=P_{n} \cdot e_{k}\left(J_{1}, J_{3}, \ldots, J_{2 n-1}\right)$.

Now the fundamental theorem on symmetric polynomials gives

Corollary 3.3. For any symmetric function $F$ and positive integer $n$,

$$
F\left(J_{1}, J_{3}, \ldots, J_{2 n-1}\right) \cdot P_{n}=P_{n} \cdot F\left(J_{1}, J_{3}, \ldots, J_{2 n-1}\right),
$$

which belongs to $\mathcal{H}_{n}$.

We are interested in the expansion of $F\left(J_{1}, J_{3}, \ldots, J_{2 n-1}\right) \cdot P_{n}$ with respect to basis $\omega^{\lambda}$ 's or $\phi_{\rho}$ 's of $\mathcal{H}_{n}$.

\section{Spherical expansion}

Our purpose in this section is to obtain the expansion of $F\left(J_{1}, J_{3}, \ldots, J_{2 n-1}\right) \cdot P_{n}$ in zonal spherical functions $\omega^{\lambda}$.

Given $F \in \mathbb{S}$ and $\lambda \vdash n$, we put

$$
F\left(A_{\lambda}^{\prime}\right):=\left.F\left(x_{1}, x_{2}, \ldots, x_{n}, 0,0, \ldots\right)\right|_{\left\{x_{1}, x_{2}, \ldots, x_{n}\right\}=A_{\lambda}^{\prime}}
$$

where $A_{\lambda}^{\prime}$ was defined in Subsection 2.1.

Theorem 4.1. For any $\lambda \vdash n$ and symmetric function $F$,

$$
F\left(J_{1}, J_{3}, \ldots, J_{2 n-1}\right) \cdot \omega^{\lambda}=\omega^{\lambda} \cdot F\left(J_{1}, J_{3}, \ldots, J_{2 n-1}\right)=F\left(A_{\lambda}^{\prime}\right) \omega^{\lambda} .
$$


Proof. In this proof, we suppose readers are familiar with standard Young tableaux, see e.g. [Sa]. For a partition $\mu$, denote by $\operatorname{SYT}(\mu)$ the set of all standard Young tableaux of shape $\mu$. For each standard Young tableau $T \in \operatorname{SYT}(\mu)$, let $e_{T} \in \mathbb{C}\left[S_{|\mu|}\right]$ be Young's orthogonal idempotent. Their definition and properties are seen in $[\mathrm{G}$. We use well-known identities

$$
J_{k} \cdot e_{T}=e_{T} \cdot J_{k}=c\left(T_{k}\right) e_{T}, \quad \sum_{T \in \operatorname{SYT}(\mu)} e_{T}=\frac{f^{\mu}}{|\mu| !} \chi^{\mu} .
$$

Here $T_{k}$ is the box $\square=\left(i_{k}, j_{k}\right)$ in $T$ labelled by $k$ and $c\left(T_{k}\right)$ is its content $j_{k}-i_{k}$. Note $f^{\mu}=|\operatorname{STY}(\mu)|$. These identities imply that, for each $\mu \vdash 2 n$,

$$
F\left(J_{1}, J_{3}, \ldots, J_{2 n-1}\right) \cdot \chi^{\mu}=\frac{(2 n) !}{f^{\mu}} \sum_{T \in \mathrm{SYT}(\mu)} F\left(c\left(T_{1}\right), c\left(T_{3}\right), \ldots, c\left(T_{2 n-1}\right)\right) e_{T} .
$$

Let $\lambda \vdash n$. Given $S=(S[i, j])_{(i, j) \in \lambda} \in \operatorname{SYT}(\lambda)$, we define the standard Young tableau $S^{\prime}=\left(S^{\prime}[i, j]\right)_{(i, j) \in 2 \lambda} \in \operatorname{SYT}(2 \lambda)$ by

$$
S^{\prime}[i, 2 j-1]=2 S[i, j]-1, \quad S^{\prime}[i, 2 j]=2 S[i, j], \quad(i, j) \in \lambda .
$$

Here $S[i, j]$ stands for the entry of $S$ in the box $(i, j)$. For example, given $S=$\begin{tabular}{l|l}
1 & 3 \\
2 & 4
\end{tabular}$\in \operatorname{SYT}((2,2))$, we have $S^{\prime}=$\begin{tabular}{|l|l|l|l|l|l|l|l}
1 & 2 & 5 & 6 \\
\hline 3 & 4 & 7 & 8
\end{tabular}$\in \operatorname{SYT}((4,4))$. Proposition 4 in [Z] claims that, given a standard Young tableau $T$ with $2 n$ boxes, $P_{n} e_{T}$ is zero unless there is a standard tableau $S$ with $n$ boxes such that $T=S^{\prime}$. We have $c\left(S_{2 k-1}^{\prime}\right)=c^{\prime}\left(S_{k}\right)$ by the construction of $S^{\prime}$. Hence it follows by (4.1) that

$$
\begin{aligned}
& F\left(J_{1}, J_{3}, \ldots, J_{2 n-1}\right) \cdot P_{n} \cdot \chi^{2 \lambda}=P_{n} \cdot F\left(J_{1}, J_{3}, \ldots, J_{2 n-1}\right) \cdot \chi^{2 \lambda} \\
= & \frac{(2 n) !}{f^{2 \lambda}} \sum_{T \in \operatorname{SYT}(2 \lambda)} F\left(c\left(T_{1}\right), c\left(T_{3}\right), \ldots, c\left(T_{2 n-1}\right)\right) P_{n} \cdot e_{T} \\
= & \frac{(2 n) !}{f^{2 \lambda}} \sum_{S \in \operatorname{SYT}(\lambda)} F\left(c^{\prime}\left(S_{1}\right), c^{\prime}\left(S_{2}\right), \ldots, c^{\prime}\left(S_{n}\right)\right) P_{n} \cdot e_{S^{\prime}} \\
= & \frac{(2 n) !}{f^{2 \lambda}} F\left(A_{\lambda}^{\prime}\right) \sum_{S \in \operatorname{SYT}(\lambda)} P_{n} \cdot e_{S^{\prime}}=\frac{(2 n) !}{f^{2 \lambda}} F\left(A_{\lambda}^{\prime}\right) \sum_{T \in \operatorname{SYT}(2 \lambda)} P_{n} \cdot e_{T}=F\left(A_{\lambda}^{\prime}\right) P_{n} \cdot \chi^{2 \lambda} .
\end{aligned}
$$

Hence we obtain the desired formula because of $\omega^{\lambda}=\left(2^{n} n !\right)^{-1} P_{n} \cdot \chi^{2 \lambda}$.

Now we give the explicit expansion of $F\left(J_{1}, J_{3}, \ldots, J_{2 n-1}\right) \cdot P_{n}$ with respect to $\omega^{\lambda}$.

Corollary 4.2. For any symmetric function $F$, we have

$$
F\left(J_{1}, J_{3}, \ldots, J_{2 n-1}\right) \cdot P_{n}=\frac{1}{(2 n-1) ! !} \sum_{\lambda \vdash n} f^{2 \lambda} F\left(A_{\lambda}^{\prime}\right) \omega^{\lambda} .
$$

In particular, the multiplicity of the identity $\operatorname{id}_{2 n}$ in $F\left(J_{1}, J_{3}, \ldots, J_{2 n-1}\right) \cdot P_{n}$ equals

$$
\frac{1}{(2 n-1) ! !} \sum_{\lambda \vdash n} f^{2 \lambda} F\left(A_{\lambda}^{\prime}\right) \text {. }
$$


Proof. Recall (see (4.8) in [CM])

$$
P_{n}=\frac{1}{(2 n-1) ! !} \sum_{\lambda \vdash n} f^{2 \lambda} \omega^{\lambda} .
$$

The claim follows from this identity and Theorem 4.1 immediately.

\section{Double coset expansion}

\subsection{Class expansion for $m_{\lambda}\left(J_{1}, J_{2}, \ldots, J_{n}\right)$}

In this subsection, we review some results in [MN]. These should be compared with theorems given in the next subsection.

A permutation $\pi$ in $S_{n}$ is of reduced cycle-type $\mu$ if $\pi$ is of the (ordinary) cycle-type $\lambda$ and $\mu=\tilde{\lambda}$. Here, as defined, $\tilde{\lambda}$ is the reduction of $\lambda$. Let $\mathfrak{c}_{\mu}(n)$ be the sum of permutations in $S_{n}$ whose reduced cycle-types are $\mu$. The element $\mathfrak{c}_{\mu}(n)$ in $\mathbb{C}\left[S_{n}\right]$ is zero unless $|\mu|+\ell(\mu) \leq n$, and $\left\{\mathfrak{c}_{\mu}(n)|| \mu \mid+\ell(\mu) \leq n\right\}$ is a basis of the center of $\mathbb{C}\left[S_{n}\right]$.

It is well known that, for any $F \in \mathbb{S}, F\left(J_{1}, J_{2}, \ldots, J_{n}\right)$ is an element of the center of the group algebra in $\mathbb{C}\left[S_{n}\right]$, see e.g. [J, $\mathbb{M N}$. We define coefficients $L_{\mu}^{\lambda}(n)$ for the monomial symmetric function $m_{\lambda}$ via

$$
m_{\lambda}\left(J_{1}, J_{2}, \ldots, J_{n}\right)=\sum_{\mu:|\mu|+\ell(\mu) \leq n} L_{\mu}^{\lambda}(n) \mathfrak{c}_{\mu}(n) .
$$

We define a number

$$
\operatorname{RC}(\lambda)=\frac{|\lambda| !}{(|\lambda|-\ell(\lambda)+1) ! \prod_{i \geq 1} m_{i}(\lambda) !} .
$$

For convenience, we put $\mathrm{RC}(0)=1$ for the zero partition (0). We call this the refined Catalan number, see [MN, §5.1]. It is known that $\mathrm{RC}(\lambda)$ is a positive integer for any $\lambda$.

Theorem 5.1 ([MN]). Let $\lambda, \mu$ be partitions. Then the following statements hold.

1. $L_{\mu}^{\lambda}(n)$ is a polynomial in $n$.

2. $L_{\mu}^{\lambda}(n)$ is zero unless $|\lambda| \geq|\mu|$ and $|\lambda| \equiv|\mu|(\bmod 2)$.

3. If $|\lambda|=|\mu|$, then $L_{\mu}^{\lambda}=L_{\mu}^{\lambda}(n)$ is independent of $n$, and given by

$$
L_{\mu}^{\lambda}=\sum_{\left(\lambda^{(1)}, \lambda^{(2)}, \ldots\right)} \operatorname{RC}\left(\lambda^{(1)}\right) \operatorname{RC}\left(\lambda^{(2)}\right) \cdots
$$

summed over all sequences of partitions such that

$$
\lambda^{(i)} \vdash \mu_{i}(i \geq 1) \quad \text { and } \quad \lambda=\lambda^{(1)} \cup \lambda^{(2)} \cup \cdots .
$$


Define coefficients $F_{\mu}^{k}(n)$ via

$$
h_{k}\left(J_{1}, J_{2}, \ldots, J_{n}\right)=\sum_{\mu:|\mu|+\ell(\mu) \leq n} F_{\mu}^{k}(n) \mathfrak{c}_{\mu}(n),
$$

where $h_{k}$ is the complete symmetric function of degree $k$. Since $h_{k}=\sum_{\lambda \vdash k} m_{\lambda}$, we have

$$
F_{\mu}^{k}(n)=\sum_{\lambda \vdash k} L_{\mu}^{\lambda}(n)
$$

Theorem 5.2 ([MN]). For $\mu \vdash k$ we have

$$
F_{\mu}^{k}(n)=\prod_{i=1}^{\ell(\mu)} \operatorname{Cat}_{\mu_{i}} .
$$

Here Cat $_{k}=\frac{1}{k+1}\left(\begin{array}{c}2 k \\ k\end{array}\right)$ is the Catalan number.

\subsection{Double coset expansion for $m_{\lambda}\left(J_{1}, J_{3}, \ldots, J_{2 n-1}\right) \cdot P_{n}$}

Like in the case of reduced cycle-types, we prefer to reduced coset-types rather than ordinary coset-types. A permutation $\sigma \in S_{2 n}$ is of reduced coset-type $\mu$ if $\mu=\tilde{\lambda}$ and the ordinary cosettype of $\sigma$ is $\lambda \vdash n$, i.e., $\Xi_{n}(\sigma)=\lambda$. In particular, elements in $H_{n}$ are of reduced coset-type (0). If $\mu$ is the reduced coset-type of $\sigma$, we write as $\xi(\sigma)=\mu$.

Define $\psi_{\mu}(n)$ to be the sum of permutations in $S_{2 n}$ whose reduced coset-types are $\mu$. Note that $\phi_{\lambda}=\psi_{\mu}(n)$ if $\lambda \vdash n$ and $\mu=\tilde{\lambda}$, where $\phi_{\lambda}$ is defined in Subsection 2.5. We have $\psi_{\mu}(n)=0$ unless $|\mu|+\ell(\mu) \leq n$. The set $\left\{\psi_{\mu}(n)|| \mu \mid+\ell(\mu) \leq n\right\}$ forms a basis of the Hecke algebra $\mathcal{H}_{n}$.

Corollary 3.2 can be rewritten as

$$
e_{k}\left(J_{1}, J_{3}, \ldots, J_{2 n-1}\right) \cdot P_{n}=\sum_{\mu \vdash k} \psi_{\mu}(n) .
$$

We would like to generalize this formula to any monomial symmetric function $m_{\lambda}$. Define coefficients $M_{\mu}^{\lambda}(n)$ by

$$
m_{\lambda}\left(J_{1}, J_{3}, \ldots, J_{2 n-1}\right) \cdot P_{n}=\sum_{\mu} M_{\mu}^{\lambda}(n) \psi_{\mu}(n)
$$

summed over $\mu$ such that $|\mu|+\ell(\mu) \leq n$. Note that, by Corollary 4.2 , the coefficient $M_{\mu}^{\lambda}(n)$ is given by

$$
M_{\mu}^{\lambda}(n)=\frac{1}{(2 n-1) ! !} \sum_{\rho \vdash n} f^{2 \rho} \omega_{\mu+\left(1^{n-|\mu|}\right)}^{\rho} m_{\lambda}\left(A_{\rho}^{\prime}\right),
$$

where $\omega_{\nu}^{\rho}$ is a value of a zonal spherical function defined in Subsection 2.5.

The following theorem is our main result for $M_{\mu}^{\lambda}(n)$.

Theorem 5.3. Let $\lambda, \mu$ be partitions. Then 
1. $M_{\mu}^{\lambda}(n)$ is a polynomial in $n$.

2. We have the inequality

$$
M_{\mu}^{\lambda}(n) \geq L_{\mu}^{\lambda}(n)
$$

3. $M_{\mu}^{\lambda}(n)$ is zero unless $|\lambda| \geq|\mu|$.

4. If $|\lambda|=|\mu|$, then we have $M_{\mu}^{\lambda}(n)=L_{\mu}^{\lambda}$. Here $L_{\mu}^{\lambda}$ is given in Theorem 5.1. In particular, $M_{\mu}^{\lambda}(n)$ is independent of $n$ in this case.

Define coefficients $G_{\mu}^{k}(n)$ via

$$
h_{k}\left(J_{1}, J_{3}, \ldots, J_{2 n-1}\right) \cdot P_{n}=\sum_{\mu:|\mu|+\ell(\mu) \leq n} G_{\mu}^{k}(n) \psi_{\mu}(n),
$$

or

$$
G_{\mu}^{k}(n)=\sum_{\lambda \vdash k} M_{\mu}^{\lambda}(n)
$$

Theorem 5.4. For $\mu \vdash k$, we have $G_{\mu}^{k}(n)=F_{\mu}^{k}(n)=\prod_{i=1}^{\ell(\mu)} \operatorname{Cat}_{\mu_{i}}$.

We will prove these theorems except part 1 of Theorem 5.3 in the coming section. The remaining statement will be proved in Section 8 by applying the theory of shifted symmetric functions.

\section{Proof of Theorem 5.3 and Theorem 5.4}

\subsection{Proof of part 2 of Theorem 5.3}

Recall that quantities $L_{\mu}^{\lambda}(n)$ and $M_{\mu}^{\lambda}(n)$ are defined by (5.1) and (5.5), respectively.

Let $\mu$ be a partition and let $n$ be a positive integer such that $n \geq|\mu|+\ell(\mu)$. We define a permutation $\pi_{\mu} \in S_{n}$ and a pair partition $\mathfrak{m}_{\mu} \in \mathcal{M}(2 n)$ as follows.

$$
\begin{aligned}
\pi_{\mu}= & \left(12 \ldots \mu_{1}+1\right)\left(\mu_{1}+2 \mu_{1}+3 \ldots \mu_{1}+\mu_{2}+2\right) \cdots, \\
\mathfrak{m}_{\mu}=\{ & \left\{1,2 \mu_{1}+2\right\},\{2,3\}, \ldots,\left\{2 \mu_{1}, 2 \mu_{1}+1\right\}, \\
& \left.\left\{2 \mu_{1}+3,2\left(\mu_{1}+\mu_{2}\right)+4\right\},\left\{2 \mu_{1}+4,2 \mu_{1}+5\right\}, \ldots,\left\{2\left(\mu_{1}+\mu_{2}\right)+2,2\left(\mu_{1}+\mu_{2}\right)+3\right\}, \ldots\right\} .
\end{aligned}
$$

For example, if $\mu=(2,1)$, we have

$$
\begin{aligned}
\pi_{(2,1)} & =(123)(45)(6)(7) \cdots(n) \\
\mathfrak{m}_{(2,1)} & =\{\{1,6\},\{2,3\},\{4,5\},\{7,10\},\{8,9\},\{10,11\}, \ldots,\{2 n-1,2 n\}\} .
\end{aligned}
$$

By construction, the reduced cycle-type of $\sigma_{\mu}$ is $\mu$ and the reduced coset-type of $\mathfrak{m}_{\mu}$ is also: $\xi\left(\mathfrak{m}_{\mu}\right)=\mu$. Note that $\mathfrak{m}_{(0)}=\{\{1,2\},\{3,4\}, \ldots,\{2 n-1,2 n\}\}$.

Define the action $\mathfrak{L}$ of $S_{2 n}$ on $\mathcal{M}(2 n)$ by

$$
\mathfrak{L}(\sigma) \mathfrak{m}=\{\{\sigma(\mathfrak{m}(1)), \sigma(\mathfrak{m}(2))\}, \ldots \ldots,\{\sigma(\mathfrak{m}(2 n-1)), \sigma(\mathfrak{m}(2 n))\}\}, \quad\left(\sigma \in S_{2 n}, \mathfrak{m} \in \mathcal{M}(2 n)\right) .
$$

Note $\mathfrak{L}(\sigma) \mathfrak{m}_{(0)}=\mathfrak{m}$ if and only if $\sigma \in \mathfrak{m} H_{n}$. 
Lemma 6.1. Let $\lambda, \mu$ be partitions and let $n \geq|\mu|+\ell(\mu)$. Fix $\mathfrak{l} \in \mathcal{M}(2 n)$ of reduced coset-type $\mu$. (In particular, we may take $\mathfrak{l}=\mathfrak{m}_{\mu}$.) Then we have

$$
M_{\mu}^{\lambda}(n)=\sum_{\substack{\sigma \in S_{2 n} \\ \mathfrak{L}(\sigma) \mathfrak{m}_{(0)}=\mathfrak{l}}}[\sigma] m_{\lambda}\left(J_{1}, J_{3}, \ldots, J_{2 n-1}\right),
$$

where $[\sigma] w$ denotes the multiplicity of $\sigma$ in $w \in \mathbb{C}\left[S_{2 n}\right]$. In particular, $M_{\mu}^{\lambda}(n)$ is a non-negative integer.

Proof. From the coset decomposition (2.5), we have

$$
\begin{aligned}
m_{\lambda}\left(J_{1}, J_{3}, \ldots, J_{2 n-1}\right) \cdot P_{n} & =\sum_{\sigma \in S_{2 n}}\left([\sigma] m_{\lambda}\left(J_{1}, J_{3}, \ldots, J_{2 n-1}\right)\right) \sigma P_{n} \\
& =\sum_{\mathfrak{m} \in \mathcal{M}(2 n)} \sum_{\sigma \in \mathfrak{m} H_{n}}\left([\sigma] m_{\lambda}\left(J_{1}, J_{3}, \ldots, J_{2 n-1}\right)\right) \mathfrak{m} P_{n} \\
& =\sum_{\mu} \sum_{\substack{\mathfrak{m} \in \mathcal{M}(2 n) \\
\xi(\mathfrak{m})=\mu}} \sum_{\sigma \in \mathfrak{m} H_{n}}\left([\sigma] m_{\lambda}\left(J_{1}, J_{3}, \ldots, J_{2 n-1}\right)\right) \mathfrak{m} P_{n} .
\end{aligned}
$$

Since

$$
\psi_{\mu}(n)=\sum_{\substack{\sigma \in S_{2 n} \\ \xi(\sigma)=\mu}} \sigma=\sum_{\substack{\mathfrak{m} \in \mathcal{M}(2 n) \\ \xi(\mathfrak{m})=\mu}} \mathfrak{m} P_{n}
$$

it follows from (5.5) that for each $\mu$,

$$
\sum_{\substack{\mathfrak{m} \in \mathcal{M}(2 n) \\ \xi(\mathfrak{m})=\mu}} \sum_{\sigma \in \mathfrak{m} H_{n}}\left([\sigma] m_{\lambda}\left(J_{1}, J_{3}, \ldots, J_{2 n-1}\right)\right) \mathfrak{m} P_{n}=M_{\mu}^{\lambda}(n) \sum_{\substack{\mathfrak{m} \in \mathcal{M}(2 n) \\ \xi(\mathfrak{m})=\mu}} \mathfrak{m} P_{n}
$$

so that $M_{\mu}^{\lambda}(n)=\sum_{\sigma \in \mathfrak{l} H_{n}}[\sigma] m_{\lambda}\left(J_{1}, J_{3}, \ldots, J_{2 n-1}\right)$. This implies the desired claim.

Let $\left(i_{1}, \ldots, i_{k}\right)$ be a weakly increasing sequence of $k$ positive integers. The sequence $\left(i_{1}, \ldots, i_{k}\right)$ is of type $\lambda \vdash k$ if $\lambda=\left(\lambda_{1}, \lambda_{2}, \ldots\right)$ is a permutation of $\left(b_{1}, b_{2}, \ldots\right)$, where $b_{p}$ is the multiplicity of $p$ in $\left(i_{1}, \ldots, i_{k}\right)$. For $\lambda \vdash k$, the monomial symmetric polynomial is expressed as

$$
m_{\lambda}\left(x_{1}, x_{2}, \ldots, x_{n}\right)=\sum_{\substack{1 \leq t_{1} \leq t_{2} \leq \cdots \leq t_{k} \leq n \\\left(t_{1}, t_{2}, \ldots, t_{k}\right): \text { type } \lambda}} x_{t_{k}} \cdots x_{t_{2}} x_{t_{1}} .
$$

Given partitions $\lambda, \mu$ with $|\lambda|=k$, we denote by $A_{n}(\lambda, \mu)$ the set of sequences $\left(u_{1}, v_{1}, u_{2}, v_{2}, \ldots, u_{k}, v_{k}\right)$ of positive integers, satisfying the following conditions:

- $\left(v_{1}, v_{2}, \ldots, v_{k}\right)$ is of type $\lambda$ and $2 \leq v_{1} \leq v_{2} \leq \cdots \leq v_{k} \leq n$;

- $u_{i}<v_{i}$ for all $1 \leq i \leq k$;

- The product of transpositions $\left(u_{k} v_{k}\right) \cdots\left(u_{2} v_{2}\right)\left(u_{1} v_{1}\right)$ coincides with $\pi_{\mu}$. 
We also denote by $B_{n}(\lambda, \mu)$ the set of the sequences $\left(s_{1}, t_{1}, \ldots, s_{k}, t_{k}\right)$ satisfying

- $\left(t_{1}, t_{2}, \ldots, t_{k}\right)$ consists of odd numbers and is of type $\lambda$, and $3 \leq t_{1} \leq t_{2} \leq \cdots \leq t_{k} \leq 2 n-1$;

- $s_{i}<t_{i}$ for all $1 \leq i \leq k$;

- $\mathfrak{L}\left(\left(s_{k} t_{k}\right) \cdots\left(s_{2} t_{2}\right)\left(s_{1} t_{1}\right)\right) \mathfrak{m}_{(0)}=\mathfrak{m}_{\mu}$.

By the definitions of $L_{\mu}^{\lambda}(n)$ and Lemma 6.1, we have

$$
L_{\mu}^{\lambda}(n)=\left|A_{n}(\lambda, \mu)\right|, \quad M_{\mu}^{\lambda}(n)=\left|B_{n}(\lambda, \mu)\right| .
$$

Now the map

$$
\left(u_{1}, v_{1}, u_{2}, v_{2}, \ldots, u_{k}, v_{k}\right) \mapsto\left(2 u_{1}-1,2 v_{1}-1,2 u_{2}-1,2 v_{2}-1, \ldots, 2 u_{k}-1,2 v_{k}-1\right)
$$

gives an injection from $A_{n}(\lambda, \mu)$ to $B_{n}(\lambda, \mu)$. Indeed, suppose $\left(u_{1}, v_{1}, \ldots, u_{k}, v_{k}\right)$ is an element of $A_{n}(\lambda, \mu)$. Then $\sigma:=\left(2 u_{k}-12 v_{k}-1\right) \cdots\left(2 u_{1}-12 v_{1}-1\right)$ permutes only odd-numbered letters, and $\sigma(2 j-1)=2 \pi_{\mu}(j)-1$ for any $j$. Since $\pi_{\mu}$ has the cycle $\left(12 \ldots \mu_{1}+1\right)$, the perfect matching $\mathfrak{L}(\sigma) \mathfrak{m}_{(0)}$ has blocks $\{3,2\},\{5,4\}, \ldots,\left\{2 \mu_{1}+1,2 \mu_{1}\right\}$ and $\left\{1,2\left(\mu_{1}+1\right)\right\}$, which are the first $\mu_{1}+1$ blocks of $\mathfrak{m}_{\mu}$. Thus, we obtain $\mathfrak{L}(\sigma) \mathfrak{m}_{(0)}=\mathfrak{m}_{\mu}$.

This injection gives $\left|A_{n}(\lambda, \mu)\right| \leq\left|B_{n}(\lambda, \mu)\right|$, that is, $L_{\mu}^{\lambda}(n) \leq M_{\mu}^{\lambda}(n)$.

\subsection{Proof of part 3 of Theorem 5.3}

The discussion in this subsection is parallel to [MN, §5.3].

From now, we suppose that $n$ is sufficiently large. In Subsection 2.4, we consider the inclusion $\mathcal{M}(2 n-2) \subset \mathcal{M}(2 n)$. Under this inclusion, the reduced coset-types are invariant.

Given $\mathfrak{m} \in \mathcal{M}(2 n)$, we define the set $\mathcal{S}(\mathfrak{m})$ by

$$
\mathcal{S}(\mathfrak{m})=\{j \in\{1,2, \ldots, n\} \mid\{\mathfrak{m}(2 j-1), \mathfrak{m}(2 j)\} \neq\{2 k-1,2 k\} \text { for any } k \geq 1\} .
$$

If the reduced coset-type of $\mathfrak{m}$ is $\mu$, then $|\mathcal{S}(\mathfrak{m})|=|\mu|+\ell(\mu)$.

For a real number $x$, we put $\lceil x\rceil=\min \{n \in \mathbb{Z} \mid x \leq n\}$. Given a positive integer $s$, define $s^{\circ}$ by

$$
s^{\circ}= \begin{cases}s+1 & \text { if } s \text { is odd, } \\ s-1 & \text { if } s \text { is even } .\end{cases}
$$

Equivalently, $s^{\circ}$ is the unique integer satisfying $\left\{s, s^{\circ}\right\} \in \mathfrak{m}_{(0)}$. We have $s=t^{\circ}$ if and only if $t=s^{\circ}$.

We use the notations in Example 2.1. Given $\mathfrak{m} \in \mathcal{M}(2 n)$ and integers $1 \leq i<j \leq 2 n$, the symbol $i \Leftrightarrow j$ stands for $\{i, j\} \in \mathfrak{m}$. Also, $i \leftrightarrow j$ stands for $j=i^{\circ}$. A part of a component of the graph $\Gamma(\mathfrak{m})$

$$
i_{1} \leftrightarrow i_{2} \Leftrightarrow i_{3} \leftrightarrow \ldots
$$

is called a piece of $\Gamma(\mathfrak{m})$. For example, $1 \leftrightarrow 2 \Leftrightarrow 6 \leftrightarrow 5$ is a piece of $\Gamma(\{\{1,4\},\{2,6\},\{3,5\}\})$. If $A$ is an empty piece, the piece $i \Leftrightarrow A \Leftrightarrow j$ stands for the piece $i \Leftrightarrow j$ simply. 
Lemma 6.2. Given an $\mathfrak{m} \in \mathcal{M}(2 n)$ and transposition $(s t)$, let $\mathfrak{n}=\mathfrak{L}((s t)) \mathfrak{m}$. Suppose that $\lambda=\left(\lambda_{1}, \lambda_{2}, \ldots\right)$ and $\mu=\left(\mu_{1}, \mu_{2}, \ldots\right)$ are the reduced coset-types of $\mathfrak{m}$ and $\mathfrak{n}$, respectively. Then either $|\mu|=|\lambda|-1,|\mu|=|\lambda|+1$, or $\mu=\lambda$ holds. Furthermore, if $|\mu|=|\lambda|+1$, then $\mathcal{S}(\mathfrak{n})=\mathcal{S}(\mathfrak{m}) \cup\left\{\left\lceil\frac{s}{2}\right\rceil,\left\lceil\frac{t}{2}\right\rceil\right\}$, and vertices $s, t$ belong to the same component of $\Gamma(\mathfrak{n})$.

Proof. First, suppose $\left\lceil\frac{s}{2}\right\rceil=\left\lceil\frac{t}{2}\right\rceil$, i.e., $t=s^{\circ}$. There exist (possibly empty) pieces $A, B$ such that $A \Leftrightarrow s \leftrightarrow t \Leftrightarrow B$ is a piece of $\Gamma(\mathfrak{m})$, and then $\Gamma(\mathfrak{n})$ has the piece $A \Leftrightarrow t \leftrightarrow s \Leftrightarrow B$. Therefore we have $\lambda=\mu$.

From now, we suppose $\left\lceil\frac{s}{2}\right\rceil \neq\left\lceil\frac{t}{2}\right\rceil$, and so $s, s^{\circ}, t$, and $t^{\circ}$ are distinct. Then the following five cases may occur:

(i) $\left|\mathcal{S}(\mathfrak{m}) \cap\left\{\left\lceil\frac{s}{2}\right\rceil,\left\lceil\frac{t}{2}\right\rceil\right\}\right|=0$.

(ii) $\left|\mathcal{S}(\mathfrak{m}) \cap\left\{\left\lceil\frac{s}{2}\right\rceil,\left\lceil\frac{t}{2}\right\rceil\right\}\right|=1$.

(iii) $\left\{\left\lceil\frac{s}{2}\right\rceil,\left\lceil\frac{t}{2}\right\rceil\right\} \subset \mathcal{S}(\mathfrak{m})$ and $s, t$ belong to different components of $\Gamma(\mathfrak{m})$.

(iv) $\Gamma(\mathfrak{m})$ has a component of the form

$$
s \leftrightarrow s^{\circ} \Leftrightarrow A \Leftrightarrow t \leftrightarrow t^{\circ} \Leftrightarrow B \Leftrightarrow s .
$$

(v) $\Gamma(\mathfrak{m})$ has a component of the form

$$
s \leftrightarrow s^{\circ} \Leftrightarrow C \Leftrightarrow t^{\circ} \leftrightarrow t \Leftrightarrow D \Leftrightarrow s .
$$

Here $A, B, C$ and $D$ are possibly empty pieces. For each case, we shall compare $\Gamma(\mathfrak{n})=$ $\Gamma(\mathcal{L}(s t) \mathfrak{m})$ with $\Gamma(\mathfrak{m})$.

In the case (i), the graph $\Gamma(\mathfrak{m})$ has components $s \leftrightarrow s^{\circ} \Leftrightarrow s$ and $t \leftrightarrow t^{\circ} \Leftrightarrow t$, and the graph $\Gamma(\mathfrak{n})$ has the new component $s \leftrightarrow s^{\circ} \Leftrightarrow t \leftrightarrow t^{\circ} \Leftrightarrow s$. Thus $\mu=\lambda \cup(1)$.

In the case (ii), we may suppose $\mathcal{S}(\mathfrak{m}) \cap\left\{\left\lceil\frac{s}{2}\right\rceil,\left\lceil\frac{t}{2}\right\rceil\right\}=\left\{\left\lceil\frac{s}{2}\right\rceil\right\}$. A piece $A \Leftrightarrow s \leftrightarrow s^{\circ} \Leftrightarrow B$ of $\Gamma(\mathfrak{m})$ with some pieces $A, B$ becomes the piece $A \Leftrightarrow t \Leftrightarrow t^{\circ} \Leftrightarrow s \leftrightarrow s^{\circ} \Leftrightarrow B$ of $\Gamma(\mathfrak{n})$. Therefore $\mu$ has a part equal to $\lambda_{j}+1$. In particular, $\mathcal{S}(\mathfrak{n})=\mathcal{S}(\mathfrak{m}) \cup\left\{\left\lceil\frac{s}{2}\right\rceil,\left\lceil\frac{t}{2}\right\rceil\right\}$.

In case (iii), $\Gamma(\mathfrak{m})$ has two components of the forms $s \leftrightarrow s^{\circ} \Leftrightarrow A \Leftrightarrow s$ and $t \leftrightarrow t^{\circ} \Leftrightarrow B \Leftrightarrow t$, where $A, B$ are non-empty pieces. Then $\Gamma(\mathfrak{n})$ has the combined component

$$
s \leftrightarrow s^{\circ} \Leftrightarrow A \Leftrightarrow t \leftrightarrow t^{\circ} \Leftrightarrow B \Leftrightarrow s .
$$

Therefore a certain part $\mu_{m}$ of $\mu$ equals $\lambda_{i}+\lambda_{j}+1$ for some $1 \leq i<j \leq \ell(\lambda)$. We also see that $\left\{\left\lceil\frac{s}{2}\right\rceil,\left\lceil\frac{t}{2}\right\rceil\right\} \subset \mathcal{S}(\mathfrak{m})=\mathcal{S}(\mathfrak{n})$.

In case (iv), $\Gamma(\mathfrak{n})$ has divided components

$$
s \leftrightarrow s^{\circ} \Leftrightarrow A \Leftrightarrow s \quad \text { and } \quad t \leftrightarrow t^{\circ} \Leftrightarrow B \Leftrightarrow t .
$$

Therefore there are $\mu_{i}$ and $\mu_{j}$ equal to $r-1$ and $\lambda_{m}-r$ for some $\lambda_{m}$ and $1 \leq r \leq \lambda_{m}$. In particular, $|\mu|=|\lambda|-1$.

In case $(\mathrm{v}), \Gamma(\mathfrak{n})$ has the component

$$
s \leftrightarrow s^{\circ} \Leftrightarrow C \Leftrightarrow t^{\circ} \leftrightarrow t \Leftrightarrow D^{\vee} \Leftrightarrow s .
$$


Here, if $D$ is the piece $i_{1} \leftrightarrow i_{2} \Leftrightarrow \cdots \leftrightarrow i_{2 p}$ then $D^{\vee}$ is the piece $i_{2 p} \leftrightarrow \cdots \Leftrightarrow i_{2} \leftrightarrow i_{1}$. In this case, $\lambda=\mu$.

For the only cases (i), (ii), and (iii), we have $|\mu|=|\lambda|+1$. The rest of the claims are already seen.

Corollary 6.3. Let $\mu$ be the reduced coset-type of $\mathfrak{m} \in \mathcal{M}(2 n)$. Suppose that there exist $p$ transpositions $\left(s_{1} t_{1}\right), \ldots,\left(s_{p} t_{p}\right)$ satisfying $\mathfrak{L}\left(\left(s_{p} t_{p}\right) \cdots\left(s_{1} t_{1}\right)\right) \mathfrak{m}_{(0)}=\mathfrak{m}$. Then $|\mu| \leq p$.

Corollary 6.4. Let $\mu \vdash p$ and let $\mathfrak{m} \in \mathcal{M}(2 n)$ be of reduced coset-type $\mu$. Suppose that there exist $p$ transpositions $\left(s_{1} t_{1}\right), \ldots,\left(s_{p} t_{p}\right)$ satisfying $\mathfrak{L}\left(\left(s_{p} t_{p}\right) \cdots\left(s_{1} t_{1}\right)\right) \mathfrak{m}_{(0)}=\mathfrak{m}$. Then $\mathcal{S}(\mathfrak{m})=\left\{\left\lceil\frac{s_{1}}{2}\right\rceil,\left\lceil\frac{t_{1}}{2}\right\rceil, \ldots,\left\lceil\frac{s_{p}}{2}\right\rceil,\left\lceil\frac{t_{p}}{2}\right\rceil\right\}$. Furthermore, for each $i$, the vertices $s_{i}, t_{i}$ belong to the same component of $\Gamma(\mathfrak{m})$.

Since $m_{\lambda}\left(J_{1}, J_{3}, \ldots, J_{2 n-1}\right)$ is a sum of products of $|\lambda|$ transpositions, part 3 of Theorem 5.3 follows from Corollary 6.3 together with Lemma 6.1.

\subsection{Proof of Theorem 5.4}

Recall that quantities $F_{\mu}^{k}(n)$ and $G_{\mu}^{k}(n)$ are defined by (5.3) and (5.8), respectively.

Let $\mathfrak{m}, \mathfrak{n} \in \mathcal{M}(2 n)$ and suppose $\mathcal{S}(\mathfrak{m}) \cap \mathcal{S}(\mathfrak{n})=\emptyset$. Denote by $\tilde{\mathfrak{m}}$ the perfect matching on $\bigsqcup_{i \in \mathcal{S}(\mathfrak{m})}\{2 i-1,2 i\}$ obtained as the union of non-trivial blocks of $\mathfrak{m}$. Clearly, $\mathcal{S}(\mathfrak{m})=\mathcal{S}(\tilde{\mathfrak{m}})$. We define the new perfect matching $\mathfrak{m} \cup \mathfrak{n} \in \mathcal{M}(2 n)$ by

$$
\mathfrak{m} \cup \mathfrak{n}=\tilde{\mathfrak{m}} \sqcup \tilde{\mathfrak{n}} \sqcup\{\{2 i-1,2 i\} \mid i \notin \mathcal{S}(\mathfrak{m}) \sqcup \mathcal{S}(\mathfrak{n})\} .
$$

If $\lambda$ and $\mu$ is the reduced coset-type of $\mathfrak{m}$ and $\mathfrak{n}$, respectively, then the reduced coset-type of $\mathfrak{m} \cup \mathfrak{n}$ is $\lambda \cup \mu$.

Example 6.1. For

$$
\begin{aligned}
\mathfrak{m} & =\{\{1,5\},\{3,4\},\{2,6\},\{7,8\},\{9,10\},\{11,12\}, \ldots,\{2 n-1,2 n\}\}, \\
\mathfrak{n} & =\{\{1,2\},\{3,4\},\{5,6\},\{7,10\},\{8,9\},\{11,12\}, \ldots,\{2 n-1,2 n\}\},
\end{aligned}
$$

we have

$$
\mathfrak{m} \cup \mathfrak{n}=\{\{1,5\},\{2,6\},\{3,4\},\{7,10\},\{8,9\},\{11,12\}, \ldots,\{2 n-1,2 n\}\} .
$$

The reduced coset-types of $\mathfrak{m}, \mathfrak{n}$, and $\mathfrak{m} \cup \mathfrak{n}$ are $(1),(1)$, and $(1,1)$, respectively.

Lemma 6.5. Let $\mathfrak{n}^{(1)}, \mathfrak{n}^{(2)} \in \mathcal{M}(2 n)$ such that $k<l$ for all $k \in \mathcal{S}\left(\mathfrak{n}^{(1)}\right)$ and $l \in \mathcal{S}\left(\mathfrak{n}^{(2)}\right)$. Suppose that the reduced coset-types of $\mathfrak{n}^{(i)}$ have sizes $r_{i} \quad(i=1,2)$. Also, there exist $r$ transpositions $\left(s_{1} t_{1}\right), \ldots,\left(s_{r} t_{r}\right)$ satisfying $\mathfrak{L}\left(\left(s_{r} t_{r}\right) \cdots\left(s_{1} t_{1}\right)\right) \mathfrak{m}_{(0)}=\mathfrak{n}^{(1)} \cup \mathfrak{n}^{(2)}$, where $r=r_{1}+r_{2}, s_{i}<t_{i}(1 \leq$ $i \leq r)$, and $t_{r} \geq \cdots \geq t_{1}$. Then

$$
\mathfrak{n}^{(1)}=\mathfrak{L}\left(\left(s_{r_{1}} t_{r_{1}}\right) \cdots\left(s_{1} t_{1}\right)\right) \mathfrak{m}_{(0)}, \quad \mathfrak{n}^{(2)}=\mathfrak{L}\left(\left(s_{r} t_{r}\right) \cdots\left(s_{r_{1}+1} t_{r_{1}+1}\right)\right) \mathfrak{m}_{(0)}
$$

and

$$
\mathcal{S}\left(\mathfrak{n}^{(1)}\right)=\left\{\left\lceil\frac{s_{1}}{2}\right\rceil,\left\lceil\frac{t_{1}}{2}\right\rceil, \ldots,\left\lceil\frac{s_{r_{1}}}{2}\right\rceil,\left\lceil\frac{t_{r_{1}}}{2}\right\rceil\right\}, \quad \mathcal{S}\left(\mathfrak{n}^{(2)}\right)=\left\{\left\lceil\frac{s_{r_{1}+1}}{2}\right\rceil,\left\lceil\frac{t_{r_{1}+1}}{2}\right\rceil, \ldots,\left\lceil\frac{s_{r}}{2}\right\rceil,\left\lceil\frac{t_{r}}{2}\right\rceil\right\} .
$$


Proof. By Corollary 6.4, we see $\mathcal{S}\left(\mathfrak{n}^{(1)}\right) \sqcup \mathcal{S}\left(\mathfrak{n}^{(2)}\right)=\mathcal{S}\left(\mathfrak{n}^{(1)} \cup \mathfrak{n}^{(2)}\right)=\left\{\left\lceil\frac{s_{1}}{2}\right\rceil,\left\lceil\frac{t_{1}}{2}\right\rceil, \ldots,\left\lceil\frac{s_{r}}{2}\right\rceil,\left\lceil\frac{t_{r}}{2}\right\rceil\right\}$. Since $t_{i}$ are not decreasing, there exists an integer $p$ such that $\left\{\left\lceil\frac{t_{1}}{2}\right\rceil, \ldots,\left\lceil\frac{t_{p}}{2}\right\rceil\right\} \subset \mathcal{S}\left(\mathfrak{n}^{(1)}\right)$ and $\left\{\left\lceil\frac{t_{p+1}}{2}\right\rceil, \ldots,\left\lceil\frac{t_{r}}{2}\right\rceil\right\} \subset \mathcal{S}\left(\mathfrak{n}^{(2)}\right)$. Furthermore, applying Corollary 6.4 again, we see that $s_{i}, t_{i}$ belong to the same component of $\Gamma\left(\mathfrak{n}^{(1)} \cup \mathfrak{n}^{(2)}\right)$, and so that $\mathcal{S}\left(\mathfrak{n}^{(1)}\right)=\left\{\left\lceil\frac{s_{1}}{2}\right\rceil,\left\lceil\frac{t_{1}}{2}\right\rceil, \ldots,\left\lceil\frac{s_{p}}{2}\right\rceil,\left\lceil\frac{t_{p}}{2}\right\rceil\right\}$ and $\mathcal{S}\left(\mathfrak{n}^{(2)}\right)=\left\{\left\lceil\frac{s_{p+1}}{2}\right\rceil,\left\lceil\frac{t_{p+1}}{2}\right\rceil, \ldots,\left\lceil\frac{s_{r}}{2}\right\rceil,\left\lceil\frac{t_{r}}{2}\right\rceil\right\}$.

Put $\rho^{(1)}=\left(s_{p} t_{p}\right) \cdots\left(s_{1} t_{1}\right)$ and $\rho^{(2)}=\left(s_{r} t_{r}\right) \cdots\left(s_{p+1} t_{p+1}\right)$. Since $\left\{s_{1}, t_{1}, \ldots, s_{p}, t_{p}\right\} \cap$ $\left\{s_{p+1}, t_{p+1}, \ldots, s_{r}, t_{r}\right\}=\emptyset$, we have $\mathfrak{n}^{(1)} \cup \mathfrak{n}^{(2)}=\mathcal{L}\left(\rho^{(2)} \rho^{(1)}\right) \mathfrak{m}_{(0)}=\mathcal{L}\left(\rho^{(1)}\right) \mathfrak{m}_{(0)} \cup \mathcal{L}\left(\rho^{(2)}\right) \mathfrak{m}_{(0)}$ and

$$
\begin{aligned}
& \mathcal{S}\left(\mathfrak{L}\left(\rho^{(1)}\right) \mathfrak{m}_{(0)}\right)=\left\{\left\lceil\frac{s_{1}}{2}\right\rceil,\left\lceil\frac{t_{1}}{2}\right\rceil, \ldots,\left\lceil\frac{s_{p}}{2}\right\rceil,\left\lceil\frac{t_{p}}{2}\right\rceil\right\}=\mathcal{S}\left(\mathfrak{n}^{(1)}\right), \\
& \mathcal{S}\left(\mathfrak{L}\left(\rho^{(2)}\right) \mathfrak{m}_{(0)}\right)=\left\{\left\lceil\frac{s_{p+1}}{2}\right\rceil,\left\lceil\frac{t_{p+1}}{2}\right\rceil, \ldots,\left\lceil\frac{s_{r}}{2}\right\rceil,\left\lceil\frac{t_{r}}{2}\right\rceil\right\}=\mathcal{S}\left(\mathfrak{n}^{(2)}\right) .
\end{aligned}
$$

Hence $\mathfrak{n}^{(i)}=\mathfrak{L}\left(\rho^{(i)}\right) \mathfrak{m}_{(0)}(i=1,2)$. In particular, the size of the reduced coset-type of $\mathfrak{L}\left(\rho^{(i)}\right) \mathfrak{m}_{(0)}$ is $r_{i} \quad(i=1,2)$. On the other hand, Corollary 6.3 and the definition of $\rho^{(i)}$ imply that $r_{1} \leq p$ and $r_{2} \leq r-p$. But $r=r_{1}+r_{2}$ so that $p=r_{1}$.

Given a positive integer $k$ and a perfect matching $\mathfrak{l} \in \mathcal{M}(2 n)$, we define $\mathcal{B}_{n}(k, \mathfrak{l})$ by the set of all sequences $\left(s_{1}, t_{1}, s_{2}, t_{2}, \ldots, s_{k}, t_{k}\right)$ of positive integers, satisfying following conditions.

- All of $t_{i}$ are odd and $3 \leq t_{1} \leq \cdots \leq t_{k} \leq 2 n-1$;

- $s_{i}<t_{i}$ for all $i$;

- $\mathfrak{L}\left(\left(s_{k} t_{k}\right) \cdots\left(s_{1} t_{1}\right)\right) \mathfrak{m}_{(0)}=\mathfrak{l}$.

Remark that the set $\mathcal{B}_{n}\left(k, \mathfrak{m}_{\mu}\right)$ coincides with the union $\bigsqcup_{\lambda \vdash k} B_{n}(\lambda, \mu)$, where $B_{n}(\lambda, \mu)$ was defined in Subsection 6.1. Also we define the set $\mathcal{B}(k, \mathfrak{l})$ as the subset of $\mathcal{B}_{n}(k, \mathfrak{l})$ which consists of sequences satisfying

$$
\left\{\left\lceil\frac{t_{1}}{2}\right\rceil, \ldots,\left\lceil\frac{t_{k}}{2}\right\rceil\right\} \subset \mathcal{S}(\mathfrak{l}) \quad \text { and } \quad t_{k}=2 a-1,
$$

where $a$ is the maximum of $\mathcal{S}(\mathfrak{l})$.

Lemma 6.6. Let $\mu \vdash k$ and let $\mathfrak{l}$ be a perfect matching of reduced coset-type $\mu$. Then $\mathcal{B}_{n}(k, \mathfrak{l})=$ $\mathcal{B}(k, \mathfrak{l})$ and $G_{\mu}^{k}(n)=|\mathcal{B}(k, \mathfrak{l})|$. In particular, both $\mathcal{B}_{n}(|\mu|, \mathfrak{l})$ and $G_{\mu}^{k}(n)$ are independent of $n$.

Proof. Let $\left(s_{1}, t_{1}, \ldots, s_{k}, t_{k}\right)$ be an element in $\mathcal{B}_{n}(k, \mathfrak{l})$. Then by Corollary 6.4, we have

$$
\left\{\left\lceil\frac{s_{1}}{2}\right\rceil,\left\lceil\frac{t_{1}}{2}\right\rceil, \ldots,\left\lceil\frac{s_{k}}{2}\right\rceil,\left\lceil\frac{t_{k}}{2}\right\rceil\right\}=\mathcal{S}(\mathfrak{l}) .
$$

Therefore $t_{k}=2 a-1$ with $a=\max \mathcal{S}(\mathfrak{l})$. Hence $\left(s_{1}, t_{1}, \ldots, s_{k}, t_{k}\right) \in \mathcal{B}(k, \mathfrak{l})$, and so $\mathcal{B}(k, \mathfrak{l})=$ $\mathcal{B}_{n}(k, \mathfrak{l})$. Also we have $G_{\mu}^{k}(n)=\left|\mathcal{B}_{n}(k, \mathfrak{l})\right|$ from Lemma 6.1.

Lemma 6.7. Let $\mu \vdash k$. Then $G_{\mu}^{k}(n)=\prod_{i=1}^{\ell(\mu)} G_{\left(\mu_{i}\right)}^{\mu_{i}}(n)$. 
Proof. We prove by induction on $\ell(\mu)=l$. If $l=1$, then our claim is trivial. Assume $l>1$.

The perfect matching $\mathfrak{m}_{\mu}$ may be uniquely expressed as $\mathfrak{m}_{\mu}=\mathfrak{m}_{\nu} \cup \mathfrak{n}$, where $\nu=\left(\mu_{1}, \mu_{2}, \ldots, \mu_{l-1}\right)$, and $\mathfrak{n}$ is the perfect matching such that $\mathcal{S}(\mathfrak{n})=\left\{\mu_{1}+\cdots+\mu_{l-1}+l+j \mid 0 \leq j \leq \mu_{l}\right\}$.

Let $\left(s_{1}, t_{1}, \ldots, s_{k}, t_{k}\right)$ be a sequence in $\mathcal{B}\left(k, \mathfrak{m}_{\mu}\right)$. By Lemma 6.5, this sequence satisfies

$$
\mathfrak{L}\left(\left(s_{k-\mu_{l}} t_{k-\mu_{l}}\right) \ldots\left(s_{1} t_{1}\right)\right)=\mathfrak{m}_{\nu}, \quad \mathfrak{L}\left(\left(s_{k} t_{k}\right) \ldots\left(s_{k-\mu_{l}+1} t_{k-\mu_{l}+1}\right)\right)=\mathfrak{n},
$$

and

$$
\begin{gathered}
3 \leq t_{1} \leq \cdots \leq t_{k-\mu_{l}}=2\left(\mu_{1}+\cdots+\mu_{l-1}+l-1\right)-1, \\
2\left(\mu_{1}+\cdots+\mu_{l-1}+l\right)+1 \leq t_{k-\mu_{l}+1} \leq \cdots \leq t_{k}=2(k+l)-1 .
\end{gathered}
$$

Therefore $\left(s_{1}, t_{1}, \ldots, s_{k-\mu_{l}}, t_{k-\mu_{l}}\right)$ belongs to $\mathcal{B}\left(k-\mu_{l}, \mathfrak{m}_{\nu}\right)$, and $\left(s_{k-\mu_{l}+1}, t_{k-\mu_{l}+1}, \ldots, t_{k}\right)$ belongs to $\mathcal{B}\left(\mu_{l}, \mathfrak{n}\right)$. This gives a bijection between $\mathcal{B}\left(k, \mathfrak{m}_{\mu}\right)$ and $\mathcal{B}\left(k-\mu_{l}, \mathfrak{m}_{\nu}\right) \times \mathcal{B}\left(\mu_{l}, \mathfrak{n}\right)$. Hence the claim follows from Lemma 6.6 and the assumption of the induction.

Lemma 6.8. $G_{(k)}^{k}(n)=$ Cat $_{k}$.

Proof. We prove by induction on $k$. Assume that for any $0 \leq q<k$ it holds that $G_{(q)}^{q}(n)=$ Cat $_{q}$. Let $\left(s_{1}, t_{1}, \ldots, s_{k}, t_{k}\right)$ be an element of $\mathcal{B}\left(k, \mathfrak{m}_{(k)}\right)$. Then $t_{k}=2 k+1$. Put $p=s_{k}$ and $\mathfrak{n}=\mathfrak{L}\left(\left(s_{k-1} t_{k-1}\right) \cdots\left(s_{1} t_{1}\right)\right) \mathfrak{m}_{(0)}$. Note that $\mathfrak{n}=\mathfrak{L}((p 2 k+1)) \mathfrak{m}_{(k)}$ and that the reduced coset-type of $\mathfrak{n}$ is of size $k-1$.

Suppose $p$ is even, say $p=2 q$ with $1 \leq q \leq k$. Since $\mathfrak{m}_{(k)}=\{\{1,2 k+2\},\{2,3\}, \ldots,\{2 k, 2 k+$ $1\}\}$, the graph $\Gamma\left(\mathfrak{L}((2 q 2 k+1)) \mathfrak{m}_{(k)}\right)$ has only one non-trivial component

$1 \leftrightarrow 2 \Leftrightarrow 3 \leftrightarrow 4 \Leftrightarrow \ldots \leftrightarrow 2 q \Leftrightarrow 2 k \leftrightarrow 2 k-1 \Leftrightarrow 2 k-2 \leftrightarrow \ldots \leftrightarrow 2 q+1 \Leftrightarrow 2 k+1 \leftrightarrow 2 k+2 \Leftrightarrow 1$.

Therefore the reduced coset-type of $\mathfrak{n}$ is $(k)$ but this is contradictory. Hence $p=s_{k}$ must be odd.

Write as $s_{k}=p=2 q-1$ with $1 \leq q \leq k$. The perfect matching $\mathfrak{n}$ can be expressed as $\mathfrak{n}=\mathfrak{n}_{q}^{\prime} \cup \mathfrak{n}_{q}^{\prime \prime}$, where $\mathfrak{n}_{q}^{\prime}$ and $\mathfrak{n}_{q}^{\prime \prime}$ are perfect matchings in $\mathcal{M}(2 n)$ such that

$$
\begin{aligned}
\widetilde{\mathfrak{n}^{\prime}} & =\{\{1,2 k+2\},\{2,3\},\{4,5\}, \ldots,\{2 q-4,2 q-3\},\{2 q-2,2 k+1\}\}, \\
\widetilde{\mathfrak{n}_{q}^{\prime \prime}} & =\{\{2 q-1,2 k\},\{2 q, 2 q+1\},\{2 q+2,2 q+3\}, \ldots,\{2 k-2,2 k-1\}\} .
\end{aligned}
$$

The reduced coset-type of $\mathfrak{n}$ is either $(q-1, k-q)$ or $(k-q, q-1)$. Therefore the sequence $\left(s_{1}, t_{1}, \ldots, s_{k-1}, t_{k-1}\right)$ belongs to $\mathcal{B}_{n}(k-1, \mathfrak{n})$. Conversely, if $\left(s_{1}^{\prime}, t_{1}^{\prime}, \ldots, s_{k-1}^{\prime}, t_{k-1}^{\prime}\right)$ is an element of $\mathcal{B}_{n}\left(k-1, \mathfrak{n}_{q}^{\prime} \cup \mathfrak{n}_{q}^{\prime \prime}\right)$, then $\left(s_{1}^{\prime}, t_{1}^{\prime}, \ldots, s_{k-1}^{\prime}, t_{k-1}^{\prime}, 2 q-1,2 k+1\right)$ belongs to $\mathcal{B}_{n}(k, \mathfrak{m})$. Therefore we have the identity

$$
G_{(k)}^{k}(n)=\left|\mathcal{B}_{n}(k, \mathfrak{m})\right|=\sum_{q=1}^{k}\left|\mathcal{B}_{n}\left(k-1, \mathfrak{n}_{q}^{\prime} \cup \mathfrak{n}_{q}^{\prime \prime}\right)\right| .
$$

It follows from Lemma 6.6. Lemma 6.7, and the induction assumption that

$$
\left|\mathcal{B}_{n}\left(k-1, \mathfrak{n}_{q}^{\prime} \cup \mathfrak{n}_{q}^{\prime \prime}\right)\right|=G_{(q-1)}^{q-1}(n) G_{(k-q)}^{k-q}(n)=\mathrm{Cat}_{q-1} \mathrm{Cat}_{k-q} .
$$

Hence the well known recurrence formula for Catalan numbers gives $G_{(k)}^{k}(n)=\sum_{q=1}^{k}$ Cat $_{q-1} \mathrm{Cat}_{k-q}=$ $\mathrm{Cat}_{k}$.

We have obtained the proof of Theorem [5.4. 


\subsection{Proof of part 4 of Theorem 5.3}

Let $\mu \vdash k$. By Theorem 5.2, part 2 of Theorem 5.3, and Theorem 5.4, we see

$$
\prod_{i=1}^{\ell(\mu)} \operatorname{Cat}_{\mu_{i}}=F_{\mu}^{k}(n)=\sum_{\lambda \vdash k} L_{\mu}^{\lambda}(n) \leq \sum_{\lambda \vdash k} M_{\mu}^{\lambda}(n)=G_{\mu}^{k}(n)=\prod_{i=1}^{\ell(\mu)} \operatorname{Cat}_{\mu_{i}}
$$

so that $M_{\mu}^{\lambda}(n)=L_{\mu}^{\lambda}(n)$ for all $\lambda \vdash k$.

\section{Weingarten functions for the orthogonal group}

Fix positive integers $N, n$ and assume $N \geq n$. We define the Weingarten function for the orthogonal group $O(N)$ by

$$
\mathrm{Wg}_{n}^{O(N)}=\frac{1}{(2 n-1) ! !} \sum_{\lambda \vdash n} \frac{f^{2 \lambda}}{\prod_{\square \in \lambda}\left(N+c^{\prime}(\square)\right)} \omega^{\lambda},
$$

which is an element of the Hecke algebra $\mathcal{H}_{n}$ of the Gelfand pair $\left(S_{2 n}, H_{n}\right)$. Here $f^{2 \lambda}$ and $c^{\prime}(\square)$ were defined in Section 2, As proved in [CM], this Weingarten function plays an important role in calculations of integrals of polynomial functions over the orthogonal group $O(N)$.

Proposition 7.1 ([CM], see also [CS]). Suppose $N \geq n$. Let $g=\left(g_{i j}\right)_{1 \leq i, j \leq N}$ be a Haardistributed random matrix from $O(N)$ and let $d g$ the normalized Haar measure on $O(N)$. Given two functions $\boldsymbol{i}, \boldsymbol{j}$ from $\{1,2, \ldots, 2 n\}$ to $\{1,2, \ldots, N\}$, we have

$$
\begin{aligned}
& \int_{g \in O(N)} g_{\boldsymbol{i}(1) \boldsymbol{j}(1)} g_{\boldsymbol{i}(2) \boldsymbol{j}(2)} \cdots g_{\boldsymbol{i}(2 n) \boldsymbol{j}(2 n)} d g \\
= & \sum_{\mathfrak{m}, \mathfrak{n} \in \mathcal{M}(2 n)} \mathrm{Wg}_{n}^{O(N)}\left(\mathfrak{m}^{-1} \mathfrak{n}\right) \prod_{k=1}^{n} \delta_{\boldsymbol{i}(\mathfrak{m}(2 k-1)), \boldsymbol{i}(\mathfrak{m}(2 k))} \delta_{\boldsymbol{j}(\mathfrak{m}(2 k-1)), \boldsymbol{j}(\mathfrak{m}(2 k))}
\end{aligned}
$$

Here we regard $\mathcal{M}(2 n)$ as a subset of $S_{2 n}$.

As a special case of Proposition 7.1, we obtain an integral expression for $\mathrm{Wg}_{n}{ }^{O(N)}(\sigma)$ :

$$
\mathrm{Wg}_{n}^{O(N)}(\sigma)=\int_{g \in O(N)} g_{1 j_{1}} g_{1 j_{2}} g_{2 j_{3}} g_{2 j_{4}} \cdots g_{n j_{2 n-1}} g_{n j_{2 n}} d g, \quad \sigma \in S_{2 n},
$$

with

$$
\left(j_{1}, j_{2}, \ldots, j_{2 n}\right)=\left(\left\lceil\frac{\sigma(1)}{2}\right\rceil,\left\lceil\frac{\sigma(2)}{2}\right\rceil, \ldots,\left\lceil\frac{\sigma(2 n)}{2}\right\rceil\right) .
$$

Remark 7.1. In Proposition 7.1, we can remove the assumption $N \geq n$. In fact, when $N<n$, it is enough to replace the range of the sum on (7.1) by partitions $\lambda \vdash n$ such that $\ell(\lambda) \leq N$. See $[\mathrm{CM}]$ for details. 
Recall that the generating function for complete symmetric polynomials $h_{k}$ is

$$
\sum_{k=0}^{\infty} h_{k}\left(x_{1}, x_{2}, \ldots, x_{n}\right) u^{k}=\prod_{i=1}^{n} \frac{1}{1-x_{i} u} .
$$

Theorem 7.2. Suppose $N \geq 2 n-1$. Then

$$
\mathrm{Wg}_{n}^{O(N)}=\sum_{k=0}^{\infty}(-1)^{k} N^{-n-k} h_{k}\left(J_{1}, J_{3}, \ldots, J_{2 n-1}\right) \cdot P_{n} .
$$

Proof. We have

$$
\begin{aligned}
\mathrm{Wg}_{n}^{O(N)} & =\frac{1}{(2 n-1) ! !} \sum_{\lambda \vdash n} f^{2 \lambda}\left(\prod_{\square \in \lambda}\left(N+c^{\prime}(\square)\right)^{-1}\right) \omega^{\lambda} \\
& =\frac{1}{(2 n-1) ! !} \sum_{\lambda \vdash n} f^{2 \lambda}\left(\sum_{k=0}^{\infty}(-1)^{k} N^{-n-k} h_{k}\left(A_{\lambda}^{\prime}\right)\right) \omega^{\lambda} \\
& =\frac{1}{(2 n-1) ! !} \sum_{k=0}^{\infty}(-1)^{k} N^{-n-k} \sum_{\lambda \vdash n} f^{2 \lambda} h_{k}\left(A_{\lambda}^{\prime}\right) \omega^{\lambda} \\
& =\sum_{k=0}^{\infty}(-1)^{k} N^{-n-k} h_{k}\left(J_{1}, J_{3}, \ldots, J_{2 n-1}\right) \cdot P_{n} .
\end{aligned}
$$

Here the second equality follows because of $\left|c^{\prime}(\square)\right| \leq 2 n-2<N$ for all $\square \in \lambda \vdash n$, and the fourth equality follows by Corollary 4.2 .

Recall that $G_{\mu}^{k}(n)$ are coefficients in

$$
h_{k}\left(J_{1}, J_{3}, \ldots, J_{2 n-1}\right) \cdot P_{n}=\sum_{\mu} G_{\mu}^{k}(n) \psi_{\mu}(n) .
$$

These coefficients appear in the asymptotic expansion of $\mathrm{Wg}_{n}^{O(N)}(\sigma)$ with respect to $\frac{1}{N}$.

Theorem 7.3. Let $\mu$ be a partition and let $N, n, k$ be positive integers. Suppose $N \geq 2 n-1$ and $n \geq|\mu|+\ell(\mu)$. For any permutation $\sigma$ in $S_{2 n}$ of reduced coset-type $\mu$, we have

$$
\begin{aligned}
\mathrm{Wg}_{n}^{O(N)}(\sigma) & =\sum_{g=0}^{\infty}(-1)^{|\mu|+g} G_{\mu}^{|\mu|+g}(n) N^{-n-|\mu|-g} \\
& =(-1)^{|\mu|} \prod_{i=1}^{\ell(\mu)} \mathrm{Cat}_{\mu_{i}} \cdot N^{-n-|\mu|}+(-1)^{|\mu|+1} \prod_{i=1}^{\ell(\mu)} G_{\mu}^{|\mu|+1}(n) \cdot N^{-n-|\mu|-1}+\cdots
\end{aligned}
$$

Proof. Theorem 7.2 and the definition of $G_{\mu}^{k}(n)$ imply

$$
\mathrm{Wg}_{n}^{O(N)}(\sigma)=\sum_{k=0}^{\infty}(-1)^{k} G_{\mu}^{k}(n) N^{-n-k} .
$$

It follows from Theorem 5.3 and Theorem 5.4 that $G_{\mu}^{k}(n)=\sum_{\lambda \vdash k} M_{\mu}^{\lambda}(n)$ is zero unless $k \geq|\mu|$ and that $G_{\mu}^{|\mu|}(n)=\prod_{i=1}^{\ell(\mu)} \mathrm{Cat}_{\mu_{i}}$. 
The unitary group version of results in this section is seen in [MN].

Collins and Śniady [CS] obtained

$$
\mathrm{Wg}_{n}^{O(N)}(\sigma)=(-1)^{|\mu|} \prod_{i \geq 1} \mathrm{Cat}_{\mu_{i}} \cdot N^{-n-|\mu|}+\mathrm{O}\left(N^{-n-|\mu|-1}\right), \quad N \rightarrow \infty,
$$

where $\sigma$ is a permutation in $S_{2 n}$ of reduced coset-type $\mu$. Our result is a refinement of their one.

We will observe the subleading coefficient $G_{\mu}^{|\mu|+1}(n)$ later, see Subsection 9.3 .

\section{Jack deformations}

A purpose in this section is to intertwine $M_{\mu}^{\lambda}(n)$ with $L_{\mu}^{\lambda}(n)$. They have been defined via symmetric functions in Jucys-Murphy elements. We define their $\alpha$-extension based on the theory of Jack polynomials.

\subsection{Jack-Plancherel measures}

Let $\alpha>0$ be a positive real number.

For each $\lambda \vdash n$, we put

$$
j_{\lambda}^{(\alpha)}=\prod_{(i, j) \in \lambda}\left\{\left(\alpha\left(\lambda_{i}-j\right)+\lambda_{j}^{\prime}-i+1\right)\left(\alpha\left(\lambda_{i}-j\right)+\lambda_{j}^{\prime}-i+\alpha\right)\right\},
$$

where $\lambda^{\prime}=\left(\lambda_{1}^{\prime}, \lambda_{2}^{\prime}, \ldots\right)$ is the conjugate partition of $\lambda$. Here the Young diagram of $\lambda^{\prime}$ is, by definition, the transpose of the Young diagram $\lambda$. Define

$$
\mathbb{P}_{n}^{(\alpha)}(\lambda)=\frac{\alpha^{n} n !}{j_{\lambda}^{(\alpha)}}
$$

This gives a probability measure on partitions of $n$ and is called the Jack-Plancherel measure or Jack measure shortly. When $\alpha=1$,

$$
\mathbb{P}_{n}^{(1)}(\lambda)=\frac{n !}{\left(H_{\lambda}\right)^{2}}=\frac{\left(f^{\lambda}\right)^{2}}{n !}
$$

where $H_{\lambda}=\sqrt{j_{\lambda}^{(1)}}$ is the product of hook-lengths of $\lambda$, and the well-known hook-length formula gives $f^{\lambda}=\frac{n !}{H_{\lambda}}$. The probability measure $\mathbb{P}_{n}^{(1)}$ is known as the Plancherel measure for the symmetric group $S_{n}$. Also, it is easy to see that

$$
\mathbb{P}_{n}^{(2)}(\lambda)=\frac{f^{2 \lambda}}{(2 n-1) ! !}, \quad \mathbb{P}_{n}^{(1 / 2)}(\lambda)=\frac{f^{\lambda \cup \lambda}}{(2 n-1) ! !} .
$$

\section{Example 8.1.}

$$
\mathbb{P}_{3}^{(\alpha)}((3))=\frac{1}{(1+\alpha)(1+2 \alpha)}, \quad \mathbb{P}_{3}^{(\alpha)}((2,1))=\frac{6 \alpha}{(2+\alpha)(1+2 \alpha)}, \quad \mathbb{P}_{3}^{(\alpha)}\left(\left(1^{3}\right)\right)=\frac{\alpha^{2}}{(1+\alpha)(2+\alpha)}
$$


The Jack-Plancherel measure has the duality relation:

$$
\mathbb{P}_{n}^{(\alpha)}(\lambda)=\mathbb{P}_{n}^{\left(\alpha^{-1}\right)}\left(\lambda^{\prime}\right),
$$

which follows from $j_{\lambda}^{(\alpha)}=\alpha^{2|\lambda|} j_{\lambda^{\prime}}^{\left(\alpha^{-1}\right)}$.

Some asymptotic properties of random variables $\lambda_{1}, \lambda_{2}, \ldots$ with repect to Jack-Plancherel measures in $n \rightarrow \infty$ are studied, see [Mat] and its references.

\subsection{Jack symmetric functions}

Recall the fundamental properties for Jack symmetric functions $J_{\lambda}^{(\alpha)}$. The details are seen in [Mac, VI-10].

Consider a scalar product on the algebra $\mathbb{S}$ of symmetric functions given by

$$
\left\langle p_{\lambda}, p_{\mu}\right\rangle_{\alpha}=\delta_{\lambda, \mu} \alpha^{\ell(\lambda)} z_{\lambda}
$$

where $z_{\lambda}$ is defined in (2.1). The Jack functions $\left\{J_{\lambda}^{(\alpha)} \mid \lambda\right.$ : partitions $\}$ are the unique family satisfying the following two conditions:

- They are of the form $J_{\lambda}^{(\alpha)}=\sum_{\mu \leq \lambda} u_{\lambda \mu}^{(\alpha)} m_{\mu}$, where each coefficient $u_{\lambda \mu}^{(\alpha)}$ is a rational function in $\alpha$, and where $\mu \leq \lambda$ stands for the dominance ordering: $|\mu|=|\lambda|$ and $\mu_{1}+\cdots+\mu_{i} \leq$ $\lambda_{1}+\cdots+\lambda_{i}$ for any $i \geq 1$.

- (orthogonality) $\left\langle J_{\lambda}^{(\alpha)}, J_{\mu}^{(\alpha)}\right\rangle_{\alpha}=\delta_{\lambda, \mu} j_{\lambda}^{(\alpha)}$ for any $\lambda, \mu$.

We note $J_{\lambda}^{(1)}=H_{\lambda} s_{\lambda}$ and $J_{\lambda}^{(2)}=Z_{\lambda}$, where $s_{\lambda}$ is a Schur function and $Z_{\lambda}$ is a zonal polynomial.

Let $\theta_{\rho}^{\lambda}(\alpha)$ be the coefficient of $p_{\rho}$ in $J_{\lambda}^{(\alpha)}$ :

$$
J_{\lambda}^{(\alpha)}=\sum_{\rho:|\rho|=|\lambda|} \theta_{\rho}^{\lambda}(\alpha) p_{\rho}
$$

By orthogonality relations for Jack and power-sum functions, we have its dual identity

$$
p_{\rho}=\alpha^{\ell(\rho)} z_{\rho} \sum_{\lambda:|\lambda|=|\rho|} \frac{\theta_{\rho}^{\lambda}(\alpha)}{j_{\lambda}^{(\alpha)}} J_{\lambda}^{(\alpha)}
$$

and the orthogonality relation for $\theta_{\rho}^{\lambda}(\alpha)$

$$
\sum_{\lambda \vdash n} \theta_{\rho}^{\lambda}(\alpha) \theta_{\pi}^{\lambda}(\alpha) \mathbb{P}_{n}^{(\alpha)}(\lambda)=\delta_{\rho \pi} \frac{\alpha^{n-\ell(\rho)} n !}{z_{\rho}} .
$$

We set $\theta_{\mu+\left(1^{n-|\mu|}\right)}^{\lambda}(\alpha)=0$ unless $|\mu|+\ell(\mu) \leq n$. Note $\theta_{\left(1^{|\lambda|}\right)}^{\lambda}(\alpha)=1$.

Let $X$ be an indeterminate. Let $\epsilon_{X}$ be the algebra homomorphism from $\mathbb{S}$ to $\mathbb{C}[X]$, defined by $\epsilon_{X}\left(p_{r}\right)=X$ for all $r \geq 1$. Then we have ([Mac, VI (10.25)])

$$
\epsilon_{X}\left(J_{\lambda}^{(\alpha)}\right)=\prod_{(i, j) \in \lambda}(X+\alpha(j-1)-(i-1)) .
$$




\subsection{Jack-Plancherel averages}

Let $A_{\lambda}^{(\alpha)}$ be the alphabet

$$
A_{\lambda}^{(\alpha)}=\{(j-1)-(i-1) / \alpha \mid(i, j) \in \lambda\}
$$

For example, $A_{(2,2)}^{(\alpha)}=\{1,0,-1 / \alpha, 1-1 / \alpha\}$. Note that $A_{\lambda}=A_{\lambda}^{(1)}$ and $A_{\lambda}^{\prime}=\left\{2 z \mid z \in A_{\lambda}^{(2)}\right\}$, which are defined in Subsection 2.1.

Given a symmetric function $F$, we define

$$
\mathcal{A}_{0}^{(\alpha)}(F, n)=\alpha^{\operatorname{deg} F} \sum_{\lambda \vdash n} F\left(A_{\lambda}^{(\alpha)}\right) \mathbb{P}_{n}^{(\alpha)}(\lambda) .
$$

More generally, for a partition $\mu$, we define

$$
\mathcal{A}_{\mu}^{(\alpha)}(F, n)=\frac{\alpha^{\operatorname{deg} F-|\mu|} z_{\mu+\left(1^{n-|\mu|}\right)}}{n !} \sum_{\lambda \vdash n} F\left(A_{\lambda}^{(\alpha)}\right) \mathbb{P}_{n}^{(\alpha)}(\lambda) \theta_{\mu+\left(1^{n-|\mu|}\right)}^{\lambda}(\alpha) .
$$

Note that $\mathcal{A}_{0}^{(\alpha)}(F, n)=\mathcal{A}_{\mu}^{(\alpha)}(F, n)$ with $\mu=(0)$.

If $F$ is homogeneous, then $F\left(A_{\lambda}^{(\alpha)}\right)=(-\alpha)^{-\operatorname{deg} F} F\left(A_{\lambda^{\prime}}^{\left(\alpha^{-1}\right)}\right)$. The $\theta_{\mu+(1|\lambda|-|\mu|)}^{\lambda}(\alpha)$ has the duality $\theta_{\mu+\left(1^{|\lambda|-|\mu|}\right)}^{\lambda}(\alpha)=(-\alpha)^{|\mu|} \theta_{\mu+\left(1^{\prime \lambda|-| \mu \mid}\right)}^{\lambda^{\prime}}\left(\alpha^{-1}\right)([\mathrm{Mac}, \mathrm{VI}(10.30)])$. Hence we have the duality relation for $\mathcal{A}_{\mu}^{(\alpha)}(F, n)$ with a homogeneous symmetric function $F$ :

$$
\mathcal{A}_{\mu}^{(\alpha)}(F, n)=(-\alpha)^{\operatorname{deg} F-|\mu|} \mathcal{A}_{\mu}^{\left(\alpha^{-1}\right)}(F, n) .
$$

The following two examples give the connection to Jucys-Murphy elements. The average $\mathcal{A}_{\mu}^{(\alpha)}(F, n)$ with $\alpha>0$ is a generalization of coefficients $L_{\mu}^{\lambda}(n), M_{\mu}^{\lambda}(n), F_{\mu}^{k}(n)$, and $G_{\mu}^{k}(n)$, which are studied in [MN] and in the first half of the present paper.

Example 8.2 $(\alpha=1)$. From $J_{\lambda}^{(1)}=H_{\lambda} s_{\lambda}$ and from the Frobenius formula $s_{\lambda}=\sum_{\rho} z_{\rho}^{-1} \chi_{\rho}^{\lambda} p_{\rho}$, we have $\theta_{\rho}^{\lambda}(1)=z_{\rho}^{-1} H_{\lambda} \chi_{\rho}^{\lambda}$, and hence

$$
\mathcal{A}_{\mu}^{(1)}(F, n)=\sum_{\lambda \vdash n} F\left(A_{\lambda}^{(1)}\right) \mathbb{P}_{n}^{(1)}(\lambda) \frac{H_{\lambda} \chi_{\mu+\left(1^{n-|\mu|}\right)}^{\lambda}(\alpha)}{n !}=\sum_{\lambda \vdash n} F\left(A_{\lambda}\right) \frac{f^{\lambda} \chi_{\mu+\left(1^{n-|\mu|}\right)}^{\lambda}}{n !} .
$$

In particular, we have $\mathcal{A}_{0}^{(1)}(F, n)=\sum_{\lambda \vdash n} F\left(A_{\lambda}^{(1)}\right) \mathbb{P}_{n}^{(1)}(\lambda)$, the average of $F\left(A_{\lambda}\right)$ with respect to the Plancherel measure $\mathbb{P}_{n}^{(1)}$. By results in [MN] (see also Subsection 5.1 in the present paper), we obtain the identity

$$
F\left(J_{1}, J_{2}, \ldots, J_{n}\right)=\sum_{\mu} \mathcal{A}_{\mu}^{(1)}(F, n) \mathfrak{c}_{\mu}(n)
$$

In particular, $L_{\mu}^{\lambda}(n)=\mathcal{A}_{\mu}^{(1)}\left(m_{\lambda}, n\right)$ and $F_{\mu}^{k}(n)=\mathcal{A}_{\mu}^{(1)}\left(h_{k}, n\right)$. 
Example $8.3(\alpha=2)$. Since $J_{\lambda}^{(2)}=Z_{\lambda}$, or since $\theta_{\rho}^{\lambda}(2)=2^{|\rho|-\ell(\rho)}|\rho| ! z_{\rho}^{-1} \omega_{\rho}^{\lambda}$, we have

$$
\mathcal{A}_{\mu}^{(2)}(F, n)=2^{\operatorname{deg} F} \sum_{\lambda \vdash n} F\left(A_{\lambda}^{(2)}\right) \mathbb{P}_{n}^{(2)}(\lambda) \omega_{\mu+\left(1^{n-|\mu|}\right)}^{\lambda}=\sum_{\lambda \vdash n} F\left(A_{\lambda}^{\prime}\right) \frac{f^{2 \lambda} \omega_{\mu+\left(1^{n-|\mu|}\right)}^{\lambda}}{(2 n-1) ! !} .
$$

Now the equation (5.6) implies $M_{\mu}^{\lambda}(n)=\mathcal{A}_{\mu}^{(2)}\left(m_{\lambda}, n\right)$ and $G_{\mu}^{k}(n)=\mathcal{A}_{\mu}^{(2)}\left(h_{k}, n\right)$. More generally,

$$
F\left(J_{1}, J_{3}, \ldots, J_{2 n-1}\right) \cdot P_{n}=\sum_{\mu} \mathcal{A}_{\mu}^{(2)}(F, n) \psi_{\mu}(n)
$$

\subsection{The $\alpha=1 / 2$ case}

We construct the $\alpha=1 / 2$ version of Example 8.2 and Example 8.3. We refer to [Mac, VII.2, Example 6,7]. Let $\epsilon$ denote the sign character of $S_{2 n}$, and let $\epsilon_{n}$ denote its restriction to $H_{n}$ : $\epsilon_{n}=\epsilon \downarrow_{H_{n}}^{S_{2 n}}$. Then $\left(S_{2 n}, H_{n}, \epsilon_{n}\right)$ is a twisted Gelfand pair in the sense of [Mac, VII.1, Example 10]. The corresponding Hecke algebra is

$$
\mathcal{H}_{n}^{\epsilon}=\left\{f: S_{2 n} \rightarrow \mathbb{C} \mid f(\zeta \sigma)=f(\sigma \zeta)=\epsilon_{n}(\zeta) f(\sigma) \quad\left(\sigma \in S_{2 n}, \zeta \in H_{n}\right)\right\} .
$$

This algebra is commutative. For each partition $\lambda \vdash n$, the $\epsilon$-spherical function $\pi^{\lambda}$ is defined by

$$
\pi^{\lambda}=\left(2^{n} n !\right)^{-1} \chi^{\lambda \cup \lambda} \cdot P_{n}^{\epsilon}=\left(2^{n} n !\right)^{-1} P_{n}^{\epsilon} \cdot \chi^{\lambda \cup \lambda},
$$

where $P_{n}^{\epsilon}=\sum_{\zeta \in H_{n}} \epsilon_{n}(\zeta) \zeta$.

For each $f \in \mathcal{H}_{n}$, let $f^{\epsilon}$ be the function on $S_{2 n}$ defined by $f^{\epsilon}(\sigma)=\epsilon(\sigma) f(\sigma)$. Then the map $f \mapsto f^{\epsilon}$ is an isomorphism of $\mathcal{H}_{n}$ to $\mathcal{H}_{n}^{\epsilon}$. Under this isomorphism, $P_{n}, \omega^{\lambda}$, and $\psi_{\mu}(n)$ are mapped to $P_{n}^{\epsilon}, \pi^{\lambda^{\prime}}$, and $\psi_{\mu}^{\epsilon}(n)=\sum_{\sigma \in H_{\mu+\left(1^{n-|\mu|}\right)}} \operatorname{sgn}(\sigma) \sigma$, respectively. Furthermore, for any homogeneous symmetric function $F$, the element $F\left(J_{1}, J_{3}, \ldots, J_{2 n-1}\right) \cdot P_{n} \in \mathcal{H}_{n}$ is mapped to $(-1)^{\operatorname{deg} F} F\left(J_{1}, J_{3}, \ldots, J_{2 n-1}\right) \cdot P_{n}^{\epsilon}$, and we have $F\left(A_{\lambda}^{\prime}\right)=(-1)^{\operatorname{deg} F} F\left(A_{\lambda^{\prime}}^{(1 / 2)}\right)$. Therefore we can obtain the following statements from facts for $F\left(J_{1}, J_{3}, \ldots, J_{2 n-1}\right) \cdot P_{n}$.

Theorem 8.1. 1. For each $0 \leq k<n$, we have

$$
e_{k}\left(J_{1}, J_{3}, \ldots, J_{2 n-1}\right) \cdot P_{n}^{\epsilon}=(-1)^{k} \sum_{\mu \vdash n} \psi_{\mu}^{\epsilon}(n) .
$$

2. For any symmetric function $F$ and partition $\lambda$ of $n$,

$$
F\left(J_{1}, J_{3}, \ldots, J_{2 n-1}\right) \cdot \pi^{\lambda}=\pi^{\lambda} \cdot F\left(J_{1}, J_{3}, \ldots, J_{2 n-1}\right)=F\left(A_{\lambda}^{(1 / 2)}\right) \pi^{\lambda} .
$$

This belongs to $\mathcal{H}_{n}^{\epsilon}$.

3. For any symmetric function $F$,

$$
F\left(J_{1}, J_{3}, \ldots, J_{2 n-1}\right) \cdot P_{n}^{\epsilon}=\frac{1}{(2 n-1) ! !} \sum_{\lambda \vdash n} f^{\lambda \cup \lambda} F\left(A_{\lambda}^{(1 / 2)}\right) \pi^{\lambda} .
$$


Furthermore, if $F$ is homogeneous, then

$$
\begin{aligned}
F\left(J_{1}, J_{3}, \ldots, J_{2 n-1}\right) \cdot P_{n}^{\epsilon} & =(-1)^{\operatorname{deg} F} \sum_{|\mu|+\ell(\mu) \leq n} \mathcal{A}_{\mu}^{(2)}(F, n) \psi_{\mu}^{\epsilon}(n) \\
& =\sum_{|\mu|+\ell(\mu) \leq n}(-1)^{|\mu|} 2^{\operatorname{deg} F-|\mu|} \mathcal{A}_{\mu}^{(1 / 2)}(F, n) \psi_{\mu}^{\epsilon}(n) .
\end{aligned}
$$

4. For any homogeneous symmetric function $F$,

$$
\mathcal{A}_{\mu}^{(1 / 2)}(F, n)=\frac{(-1)^{|\mu|}}{2^{\operatorname{deg} F-|\mu|}} \sum_{\lambda \vdash n} F\left(A_{\lambda}^{(1 / 2)}\right) \frac{f^{\lambda \cup \lambda} \omega_{\mu+\left(1^{\lambda^{\prime}-|\mu|}\right)}}{(2 n-1) ! !} .
$$

In a similar way to Section 7 , we can observe the deep connection between $F\left(J_{1}, J_{3}, \ldots, J_{2 n-1}\right)$. $P_{n}^{\epsilon}$ and integrals over symplectic groups. That connection will be seen in the forthcoming paper.

\subsection{Some properties}

Lemma 8.2. Let $F$ be a symmetric function and $n$ a positive integer. Assume that there exist complex numbers $\{a(\mu) \mid \mu$ is a partition $\}$ such that

$$
F\left(A_{\lambda}^{(\alpha)}\right)=\sum_{\mu} a(\mu) \theta_{\mu+(1|\lambda|-|\mu|)}^{\lambda}(\alpha)
$$

for any partitions $\lambda$. Then $\mathcal{A}_{\mu}^{(\alpha)}(F, n)=\alpha^{\operatorname{deg} F} a(\mu)$ for each $\mu$.

Proof. We have

$$
\mathcal{A}_{\mu}^{(\alpha)}(F, n)=\frac{\alpha^{\operatorname{deg} F-|\mu|} z_{\mu+\left(1^{n-|\mu|}\right)}}{n !} \sum_{\nu} a(\nu) \sum_{\lambda \vdash n} \mathbb{P}_{n}^{(\alpha)}(\lambda) \theta_{\mu+\left(1^{n-|\mu|}\right)}^{\lambda}(\alpha) \theta_{\nu+\left(1^{n-|\nu|}\right)}^{\lambda}(\alpha) .
$$

The claim follows from the orthogonality relation (8.4). 3.2 .

The following theorem is a Jack deformation of Jucys' result [J] and its analogue, Corollary

\section{Proposition 8.3.}

$$
\mathcal{A}_{\mu}^{(\alpha)}\left(e_{k}, n\right)= \begin{cases}1 & \text { if }|\mu|=k \\ 0 & \text { otherwise. }\end{cases}
$$

Proof. Let $X$ be an indeterminate and let $\lambda \vdash n$. It follows from (8.5) that

$$
\begin{aligned}
& \sum_{k=0}^{n} e_{k}\left(A_{\lambda}^{(\alpha)}\right) X^{k}=(X / \alpha)^{n} \prod_{(i, j) \in \lambda}(\alpha / X+\alpha(j-1)-(i-1))=(X / \alpha)^{n} \epsilon_{\alpha / X}\left(J_{\lambda}^{(\alpha)}\right) \\
= & (X / \alpha)^{n} \sum_{\rho \vdash n} \theta_{\rho}^{\lambda}(\alpha) \epsilon_{\alpha / X}\left(p_{\rho}\right)=\sum_{\rho \vdash n} \theta_{\rho}^{\lambda}(\alpha)(X / \alpha)^{n-\ell(\rho)}=\sum_{k=0}^{n} \alpha^{-k} \sum_{\nu \vdash k} \theta_{\nu+\left(1^{n-k}\right)}^{\lambda}(\alpha) X^{k},
\end{aligned}
$$


which gives

$$
e_{k}\left(A_{\lambda}^{(\alpha)}\right)=\alpha^{-k} \sum_{\nu \vdash k} \theta_{\nu+(1|\lambda|-k)}^{\lambda}(\alpha) .
$$

The claim follows from this identity and Lemma 8.2,

Remark 8.1. The equation (8.9) is seen in [L1, Theorem 5.4].

Theorem 8.4. Let $F$ be any symmetric function and $\mu$ a partition. Then $\mathcal{A}_{\mu}^{(\alpha)}(F, n)$ is a polynomial in $n$. If the expansion of $F$ in $p_{\rho}$ is given by $F=\sum_{\rho} a(\rho) p_{\rho}$, then the degree of $\mathcal{A}_{\mu}^{(\alpha)}(F, n)$ in $n$ is at most

$$
\max _{a(\rho) \neq 0}(|\rho|+\ell(\rho))-(|\mu|+\ell(\mu)) .
$$

This theorem at $\alpha=2$ with Example 8.3 implies part 1 of Theorem 5.3 . The proof is given in the next subsection by applying shifted symmetric function theory.

Example 8.4. Since the monomial symmetric function is expanded as

$$
m_{\lambda}=p_{\lambda}+\sum_{\rho>\lambda} a(\rho) p_{\rho}
$$

the degree of the polynomial $\mathcal{A}_{\mu}^{(\alpha)}\left(m_{\lambda}, n\right)$ is at most $|\lambda|+\ell(\lambda)-(|\mu|+\ell(\mu))$. But this evaluation is not sharp. Indeed, as we will observe below, the degree of $\mathcal{A}_{(0)}^{(\alpha)}\left(m_{(3)}, n\right)=\alpha(\alpha-1)\left(\begin{array}{l}n \\ 2\end{array}\right)$ is 2 but $(|\lambda|+\ell(\lambda))-(|\mu|+\ell(\mu))=4$ with $\lambda=(3)$ and $\mu=(0)$.

\subsection{Shifted symmetric functions and proof of Theorem 8.4}

Following to [KOO, L2, we review the theory of shifted symmetric functions related to Jack functions.

A polynomial in $n$ variables $x_{1}, x_{2}, \ldots, x_{n}$ is said to be shifted-symmetric if it is symmetric in the variables $y_{i}:=x_{i}-i / \alpha$. Denote by $\mathbb{S}_{\alpha}^{*}(n)$ the subalgebra of shifted-symmetric functions in $\mathbb{C}\left[x_{1}, x_{2}, \ldots, x_{n}\right]$.

Consider an infinite alphabet $x=\left(x_{1}, x_{2}, \ldots\right)$ and consider the morphism $F\left(x_{1}, x_{2}, \ldots, x_{n}, x_{n+1}\right) \mapsto$ $F\left(x_{1}, x_{2}, \ldots, x_{n}, 0\right)$ from $\mathbb{S}_{\alpha}^{*}(n+1)$ to $\mathbb{S}_{\alpha}^{*}(n)$. As the definition of $\mathbb{S}$, we can define the algebra $\mathbb{S}_{\alpha}^{*}$ as the projective limit of the sequence $\left(\mathbb{S}_{\alpha}^{*}(n)\right)_{n \geq 1}$. Elements of $\mathbb{S}_{\alpha}^{*}$ are called shifted-symmetric functions and written as $F(x)=F\left(x_{1}, x_{2}, \ldots\right)$ using infinite variables. Denote by $\operatorname{deg} F$ the degree of $F$.

For each $F \in \mathbb{S}_{\alpha}^{*}$, we may evaluate at partitions: $F(\lambda)=F\left(\lambda_{1}, \lambda_{2}, \ldots\right)$. We denote by $[F] \in \mathbb{S}$ the homogeneous symmetric terms of degree $\operatorname{deg} F$. We call $[F]$ the leading symmetric term of $F$. The map $F \mapsto[F]$ provides a canonical isomorphism of the graded algebra associated to the filtered algebra $\mathbb{S}_{\alpha}^{*}$ onto $\mathbb{S}$. Assuming that the leading terms $\left[F_{1}\right],\left[F_{2}\right], \ldots$ of a sequence $F_{1}, F_{2}, \ldots$ in $\mathbb{S}_{\alpha}^{*}$ generate the algebra $\mathbb{S}$, this sequence itself generates $\mathbb{S}_{\alpha}^{*}$.

For each integer $k \geq 1$, consider a polynomial

$$
p_{k}^{*}(x ; \alpha)=\sum_{i \geq 1}\left(\left(x_{i}-(i-1) / \alpha\right)^{\downarrow k}-(-(i-1) / \alpha)^{\downarrow k}\right)
$$


with $a^{\downarrow k}=a(a-1) \cdots(a-k+1)$. Then these polynomials are shifted-symmetric. Since $\left[p_{k}^{*}(\cdot ; \alpha)\right]=p_{k}$ and since the $p_{k}$ generate $\mathbb{S}$, they generate $\mathbb{S}_{\alpha}^{*}$.

For $F \in \mathbb{S}$ and a partition $\lambda$, we put $H_{F}^{(\alpha)}(\lambda)=F\left(A_{\lambda}^{(\alpha)}\right)$.

Lemma 8.5 (Lemma 7.1 in [L2]). For any integer $k \geq 1$, the function $\lambda \mapsto H_{p_{k}}^{(\alpha)}(\lambda)=p_{k}\left(A_{\lambda}^{(\alpha)}\right)$ defines a shifted symmetric function of degree $\operatorname{deg} H_{p_{k}}^{(\alpha)}=k+1$. Specifically,

$$
H_{p_{k}}^{(\alpha)}(\lambda)=\sum_{m=1}^{k} S(k, m) \frac{p_{m+1}^{*}(\lambda ; \alpha)}{m+1} .
$$

Here $S(k, m)$ are Stirling's numbers of second kinds, defined via $u^{k}=\sum_{m=1}^{k} S(k, m) u^{\downarrow m}$.

Since $p_{k}$ generate $\mathbb{S}$, we have the following corollary.

Corollary 8.6. For any $F \in \mathbb{S}$, the function $\lambda \mapsto H_{F}^{(\alpha)}(\lambda)$ defines a shifted symmetric function. Furthermore, if the expansion of $F$ in $p_{\rho}$ is given by $F=\sum_{\rho} a(\rho) p_{\rho}$, then the degree of $H_{F}^{(\alpha)}$ is $\max _{a(\rho) \neq 0}(|\rho|+\ell(\rho))$.

Now we define shifted Jack functions $J_{\mu}^{*}(x ; \alpha)$. They are defined by

$$
J_{\mu}^{*}(\lambda ; \alpha)=\frac{|\lambda|^{\downarrow|\mu|}\left\langle p_{1}^{|\lambda|-|\mu|} J_{\mu}^{(\alpha)}, J_{\lambda}^{(\alpha)}\right\rangle_{\alpha}}{\alpha^{|\lambda|}|\lambda| !} .
$$

Lemma $8.7([\mathrm{KOO}])$. The $J_{\mu}^{*}(x ; \alpha)$ are shifted-symmetric and satisfy the following properties.

1. $\left[J_{\mu}^{*}(\cdot ; \alpha)\right]=J_{\mu}^{(\alpha)}$. Hence the $J_{\mu}^{*}(x ; \alpha)$ form a basis of $\mathbb{S}_{\alpha}^{*}$.

2. $J_{\mu}^{*}(\lambda ; \alpha)=0$ unless $\mu_{i} \leq \lambda_{i}$ for all $i \geq 1$.

3. $J_{\mu}^{*}(\mu ; \alpha)=\alpha^{-|\mu|} j_{\mu}^{(\alpha)}$.

The following theorem is a slight extension of Theorem 5.5 in [0].

Proposition 8.8. Let $\mu, \nu$ be partitions. If $|\nu| \geq|\mu|+\ell(\mu)$, then we have

$$
\frac{z_{\mu+\left(1^{n-|\mu|}\right)}}{n !} \sum_{\lambda \vdash n} J_{\nu}^{*}(\lambda ; \alpha) \mathbb{P}_{n}^{(\alpha)}(\lambda) \theta_{\mu+\left(1^{n-|\mu|}\right)}^{\lambda}(\alpha)=\left(\begin{array}{c}
n-|\mu|-\ell(\mu) \\
|\nu|-|\mu|-\ell(\mu)
\end{array}\right) z_{\mu+\left(1^{|\nu|-|\mu|}\right)} \theta_{\mu+\left(1^{|\nu|-|\mu|}\right)}(\alpha),
$$

which is a polynomial in $n$ of degree $|\nu|-|\mu|-\ell(\mu)$. Otherwise, both sides are zero.

Proof. Put $m=|\nu|$. If $n<m$, then both sides vanish by part 2 of Lemma 8.7, and so we may 
assume $n \geq m$. We have

$$
\begin{aligned}
& \frac{z_{\mu+\left(1^{n-|\mu|}\right)}}{n !} \sum_{\lambda \vdash n} J_{\nu}^{*}(\lambda ; \alpha) \mathbb{P}_{n}^{(\alpha)}(\lambda) \theta_{\mu+\left(1^{n-|\mu|}\right)}^{\lambda}(\alpha) \\
= & \frac{z_{\mu+\left(1^{n-|\mu|}\right)}}{n !} \sum_{\lambda \vdash n} \frac{n^{\downarrow m}\left\langle p_{1}^{n-m} J_{\nu}^{(\alpha)}, J_{\lambda}^{(\alpha)}\right\rangle_{\alpha}}{j_{\lambda}^{(\alpha)}} \theta_{\mu+\left(1^{n-|\mu|}\right)}^{\lambda}(\alpha) \\
= & \frac{n^{\downarrow m}}{\alpha^{n-|\mu|} n !}\left\langle p_{1}^{n-m} J_{\nu}^{(\alpha)}, \sum_{\lambda \vdash n} \frac{\alpha^{n-|\mu|} z_{\mu+\left(1^{n-|\mu|}\right)}}{j_{\lambda}^{(\alpha)}} \theta_{\mu+\left(1^{n-|\mu|}\right)}^{\lambda} J_{\lambda}^{(\alpha)}\right\rangle_{\alpha} \\
= & \left.\frac{n^{\downarrow m}}{\alpha^{n-|\mu|} n !}\left\langle p_{1}^{n-m} J_{\nu}^{(\alpha)}, p_{\mu+\left(1^{n-|\mu|}\right)}\right\rangle_{\alpha} \quad \text { by (‥3) }\right) .
\end{aligned}
$$

Using the fact that the adjoint to the multiplication by $p_{1}$ with respect to $\langle\cdot, \cdot\rangle_{\alpha}$ is $\alpha \frac{\partial}{\partial p_{1}}$, we have

$$
=\frac{n^{\downarrow m}}{\alpha^{n-|\mu|} n !}\left\langle J_{\nu}^{(\alpha)}, \alpha^{n-m}\left(\frac{\partial}{\partial p_{1}}\right)^{n-m} p_{\mu+\left(1^{n-|\mu|}\right)}\right\rangle_{\alpha} .
$$

Since $m_{1}\left(\mu+\left(1^{n-|\mu|}\right)\right)=n-|\mu|-\ell(\mu)$, the symmetric function $\left(\frac{\partial}{\partial p_{1}}\right)^{n-m} p_{\mu+\left(1^{n-|\mu|}\right)}$ vanishes unless $m \geq|\mu|+\ell(\mu)$. If $n \geq m \geq|\mu|+\ell(\mu)$, then (8.10) equals

$$
\begin{aligned}
& =\frac{n^{\downarrow m}}{\alpha^{m-|\mu|} n !}(n-|\mu|-\ell(\mu))^{\downarrow(n-m)}\left\langle J_{\nu}^{(\alpha)}, p_{\mu+\left(1^{m-|\mu|}\right)}\right\rangle_{\alpha} \\
& =\left(\begin{array}{c}
n-|\mu|-\ell(\mu) \\
m-|\mu|-\ell(\mu)
\end{array}\right) z_{\mu+\left(1^{m-|\mu|}\right)} \theta_{\mu+\left(1^{m-|\mu|}\right)}^{\nu}(\alpha) .
\end{aligned}
$$

Remark 8.2. Proposition 8.8 can be rewritten as follows: for partitions $\nu, \mu$ such that $|\nu| \geq$ $|\mu|+\ell(\mu)$ and for any $n \geq 0$,

$$
\sum_{\lambda \vdash n} J_{\nu}^{*}(\lambda ; \alpha) \mathbb{P}_{n}^{(\alpha)}(\lambda) \theta_{\mu+\left(1^{n-|\mu|}\right)}^{\lambda}(\alpha)=n^{\downarrow|\nu|} \theta_{\mu+(1|\nu|-|\mu|)}^{\nu}(\alpha) .
$$

In particular, we obtain a simple identity

$$
\sum_{\lambda \vdash n} J_{\nu}^{*}(\lambda ; \alpha) \mathbb{P}_{n}^{(\alpha)}(\lambda)=n^{\downarrow|\nu|},
$$

which is seen in [O, Theorem 5.5]. Lassalle obtained a similar identity. Specifically, Equation (3.3) in [L3] implies that, for partitions $\nu$ and $\mu$ such that $|\nu| \geq|\mu|+\ell(\mu)$ and for any $m \geq 0$,

$$
\sum_{\rho \vdash m} J_{\rho}^{*}(\nu ; \alpha) \mathbb{P}_{m}^{(\alpha)}(\rho) \theta_{\mu+\left(1^{m-|\mu|}\right)}^{\rho}(\alpha)=\frac{(|\nu|-|\mu|-\ell(\mu)) ! m !}{(|\nu|-m) !(m-|\mu|-\ell(\mu)) !} \theta_{\mu+\left(1^{|\nu|-|\mu|}\right)}^{\nu}(\alpha) .
$$

Proof of Theorem 8.4 and part 1 of Theorem 5.3. The statement follows from Theorem 8.6, part 1 of Lemma 8.7, and Proposition 8.8. 


\section{$9 \quad$ Examples and open problems}

\subsection{Examples of $\mathcal{A}_{\mu}^{(\alpha)}(F, n)$}

We give examples of $\mathcal{A}_{\mu}^{(\alpha)}\left(m_{\lambda}, n\right)$ and $\mathcal{A}_{\mu}^{(\alpha)}\left(h_{k}, n\right)$, studied in the previous section.

$|\lambda|=0,1$.

$$
\mathcal{A}_{\mu}^{(\alpha)}\left(m_{(0)}, n\right)=\delta_{\mu,(0)} . \quad \mathcal{A}_{\mu}^{(\alpha)}\left(m_{(1)}, n\right)=\delta_{\mu,(1)} .
$$

$|\lambda|=2$

$$
\begin{aligned}
\mathcal{A}_{\mu}^{(\alpha)}\left(m_{(2)}, n\right) & =\delta_{\mu,(2)}+(\alpha-1) \delta_{\mu,(1)}+\alpha\left(\begin{array}{l}
n \\
2
\end{array}\right) \delta_{\mu,(0)} . \\
\mathcal{A}_{\mu}^{(\alpha)}\left(m_{\left(1^{2}\right)}, n\right) & =\delta_{\mu,(2)}+\delta_{\mu,\left(1^{2}\right)} . \\
\mathcal{A}_{\mu}^{(\alpha)}\left(h_{2}, n\right) & =2 \delta_{\mu,(2)}+\delta_{\mu,\left(1^{2}\right)}+(\alpha-1) \delta_{\mu,(1)}+\alpha\left(\begin{array}{l}
n \\
2
\end{array}\right) \delta_{\mu,(0)} .
\end{aligned}
$$

$|\lambda|=3$

$$
\begin{aligned}
\mathcal{A}_{\mu}^{(\alpha)}\left(m_{(3)}, n\right) & =\delta_{\mu,(3)}+3(\alpha-1) \delta_{\mu,(2)}+\left(2 \alpha n+\alpha^{2}-5 \alpha+1\right) \delta_{\mu,(1)}+\alpha(\alpha-1)\left(\begin{array}{c}
n \\
2
\end{array}\right) \delta_{\mu,(0)} . \\
\mathcal{A}_{\mu}^{(\alpha)}\left(m_{(2,1)}, n\right) & =3 \delta_{\mu,(3)}+\delta_{\mu,(2,1)}+3(\alpha-1) \delta_{\mu,(2)}+2(\alpha-1) \delta_{\mu,\left(1^{2}\right)}+\alpha\left(\left(\begin{array}{c}
n \\
2
\end{array}\right)-1\right) \delta_{\mu,(1)} . \\
\mathcal{A}_{\mu}^{(\alpha)}\left(m_{\left(1^{3}\right)}, n\right) & =\delta_{\mu,(3)}+\delta_{\mu,(2,1)}+\delta_{\mu,\left(1^{3}\right)} . \\
\mathcal{A}_{\mu}^{(\alpha)}\left(h_{3}, n\right) & =5 \delta_{\mu,(3)}+2 \delta_{\mu,(2,1)}+\delta_{\mu,\left(1^{3}\right)}+6(\alpha-1) \delta_{\mu,(2)}+2(\alpha-1) \delta_{\mu,\left(1^{2}\right)} \\
+ & \left(\frac{1}{2} \alpha n^{2}+\frac{3}{2} \alpha n+\alpha^{2}-6 \alpha+1\right) \delta_{\mu,(1)}+\alpha(\alpha-1)\left(\begin{array}{l}
n \\
2
\end{array}\right) \delta_{\mu,(0)} .
\end{aligned}
$$

In fact, the identities for $m_{\left(1^{k}\right)}$ are given by Proposition 8.3, Lassalle [L3, L4] (see also [L1, Conjecture 8.1]) gives the expansion of $\theta_{\mu+\left(1^{|\lambda|-|\mu|}\right)}^{\lambda}(\alpha)$ with respect to $p_{\rho}\left(A_{\lambda}^{(\alpha)}\right)$ : Letting $\hat{\theta}_{\mu}(\lambda)=\theta_{\mu+\left(1^{|\lambda|-|\mu|}\right)}^{\lambda}(\alpha)$ and $\hat{p}_{\rho}(\lambda)=p_{\rho}\left(A_{\lambda}^{(\alpha)}\right)$,

$$
\begin{aligned}
\hat{\theta}_{(1)}(\lambda)= & \alpha \hat{p}_{(1)}(\lambda), \\
\hat{\theta}_{(2)}(\lambda)= & \alpha^{2} \hat{p}_{(2)}(\lambda)-\alpha(\alpha-1) \hat{p}_{(1)}(\lambda)-\alpha\left(\begin{array}{c}
|\lambda| \\
2
\end{array}\right), \\
\hat{\theta}_{\left(1^{2}\right)}(\lambda)= & -\frac{3}{2} \alpha^{2} \hat{p}_{(2)}(\lambda)+\frac{1}{2} \alpha^{2} \hat{p}_{\left(1^{2}\right)}(\lambda)+\alpha(\alpha-1) \hat{p}_{(1)}(\lambda)+\alpha\left(\begin{array}{c}
|\lambda| \\
2
\end{array}\right), \\
\hat{\theta}_{(3)}(\lambda)= & \alpha^{3} \hat{p}_{(3)}(\lambda)-3 \alpha^{2}(\alpha-1) \hat{p}_{(2)}(\lambda)+\alpha\left(-2 \alpha|\lambda|+2 \alpha^{2}-\alpha+2\right) \hat{p}_{(1)}(\lambda)+2 \alpha(\alpha-1)\left(\begin{array}{c}
|\lambda| \\
2
\end{array}\right), \\
\hat{\theta}_{(2,1)}(\lambda)= & -4 \alpha^{3} \hat{p}_{(3)}(\lambda)+\alpha^{3} \hat{p}_{(2,1)}(\lambda)+9 \alpha^{2}(\alpha-1) \hat{p}_{(2)}(\lambda)-\alpha^{2}(\alpha-1) \hat{p}_{\left(1^{2}\right)}(\lambda) \\
& +\alpha\left(-\frac{\alpha}{2}|\lambda|^{2}+\frac{13 \alpha}{2}|\lambda|-5 \alpha^{2}+2 \alpha-5\right) \hat{p}_{(1)}(\lambda)-5 \alpha(\alpha-1)\left(\begin{array}{c}
|\lambda| \\
2
\end{array}\right) .
\end{aligned}
$$


Using these, we can express $p_{\rho}\left(A_{\lambda}^{(\alpha)}\right)$ in terms of $\hat{\theta}_{\mu}(\lambda)$ and therefore also $m_{\nu}\left(A_{\lambda}^{(\alpha)}\right)$. Hence our above examples follow by Lemma 8.2 .

Those examples are reduced as typical cases

$$
\begin{aligned}
L_{\mu}^{\lambda}(n) & =\mathcal{A}_{\mu}^{(1)}\left(m_{\lambda}, n\right), & F_{\mu}^{k}(n) & =\mathcal{A}_{\mu}^{(1)}\left(h_{k}, n\right), \\
M_{\mu}^{\lambda}(n) & =\mathcal{A}_{\mu}^{(2)}\left(m_{\lambda}, n\right), & G_{\mu}^{k}(n) & =\mathcal{A}_{\mu}^{(2)}\left(h_{k}, n\right) .
\end{aligned}
$$

For example, we obtain

$$
\mathcal{A}_{\mu}^{(2)}\left(h_{3}, n\right)=5 \delta_{\mu,(3)}+2 \delta_{\mu,(2,1)}+\delta_{\mu,\left(1^{3}\right)}+6 \delta_{\mu,(2)}+2 \delta_{\mu,\left(1^{2}\right)}\left(n^{2}+3 n-7\right) \delta_{\mu,(1)}+n(n-1) \delta_{\mu,(0)},
$$

or, by Example 8.3 .

$$
\begin{gathered}
h_{3}\left(J_{1}, J_{3}, \ldots, J_{2 n-1}\right) \cdot P_{n}=5 \psi_{(3)}(n)+2 \psi_{(2,1)}(n)+\psi_{\left(1^{3}\right)}(n)+6 \psi_{(2)}(n)+2 \psi_{\left(1^{2}\right)}(n) \\
+\left(n^{2}+3 n-7\right) \psi_{(1)}(n)+n(n-1) \psi_{(0)}(n) .
\end{gathered}
$$

See also the $\alpha=1$ cases in $[\mathrm{MN}, \S 8.1]$.

We remark that a conjecture for $\mathcal{A}_{0}^{(\alpha)}\left(p_{\lambda}, n\right)$ is given by [L1, Conjecture 12.1].

\subsection{Table of asymptotic expansions of $\mathrm{Wg}_{n}^{O(N)}$}

We give some examples of the expansion (7.2), by using coefficients appearing in Subsection 9.1.

Given a partition $\mu$, we define $\mathrm{Wg}^{O(N)}(\mu ; n)=\mathrm{Wg}_{n}^{O(N)}(\sigma)$, where $\sigma$ is a permutation in $S_{2 n}$ of reduced coset-type $\mu$. For example,

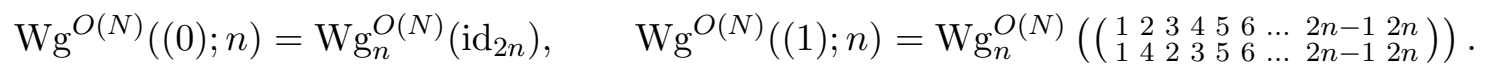

Theorem 7.3 and examples in the previous subsection with $\alpha=2$ give the following asymptotic expansions. As $N \rightarrow \infty$,

$$
\begin{aligned}
\mathrm{Wg}^{O(N)}((0) ; n) & =N^{-n}+n(n-1) N^{-n-2}-n(n-1) N^{-n-3}+\mathrm{O}\left(N^{-n-4}\right) . \\
\mathrm{Wg}^{O(N)}((1) ; n) & =-N^{-n-1}+N^{-n-2}-\left(n^{2}+3 n-7\right) N^{-n-3}+\mathrm{O}\left(N^{-n-4}\right) . \\
\mathrm{Wg}^{O(N)}((2) ; n) & =2 N^{-n-2}-6 N^{-n-3}+\mathrm{O}\left(N^{-n-4}\right) . \\
\mathrm{Wg}^{O(N)}\left(\left(1^{2}\right) ; n\right) & =N^{-n-2}-2 N^{-n-3}+\mathrm{O}\left(N^{-n-4}\right) .
\end{aligned}
$$

On the other hand, in [CM], the explicit values of $\mathrm{Wg}_{n}^{O(N)}(\sigma)$ for $n \leq 6$ are given. We remark that in $\mathrm{CM}$ ordinary coset-types were used, not reduced ones. Using a computer with the table in [CM], we obtain the following expansions.

$$
\begin{aligned}
& \mathrm{Wg}^{O(N)}((0) ; 2)=N^{-2}-0 N^{-3}+2 N^{-4}-2 N^{-5}+6 N^{-6}-10 N^{-7}+22 N^{-8}-\cdots \\
& \mathrm{Wg}^{O(N)}((0) ; 3)=N^{-3}-0 N^{-4}+6 N^{-5}-6 N^{-6}+50 N^{-7}-126 N^{-8}+610 N^{-9}-\cdots \\
& \mathrm{Wg}^{O(N)}((0) ; 4)=N^{-4}-0 N^{-5}+12 N^{-6}-12 N^{-7}+176 N^{-8}-468 N^{-9}+3544 N^{-10}-\cdots \\
& \mathrm{Wg}^{O(N)}((0) ; 5)=N^{-5}-0 N^{-6}+20 N^{-7}-20 N^{-8}+440 N^{-9}-1180 N^{-10}+12480 N^{-11}-\cdots \\
& \mathrm{Wg}^{O(N)}((0) ; 6)=N^{-6}-0 N^{-7}+30 N^{-8}-30 N^{-9}+910 N^{-10}-2430 N^{-11}+33710 N^{-12}-\cdots
\end{aligned}
$$




$$
\begin{aligned}
& \mathrm{Wg}^{O(N)}((1) ; 2)=-N^{-3}+N^{-4}-3 N^{-5}+5 N^{-6}-11 N^{-7}+21 N^{-8}-43 N^{-9}+\cdots \\
& \mathrm{Wg}^{O(N)}((1) ; 3)=-N^{-4}+N^{-5}-11 N^{-6}+29 N^{-7}-147 N^{-8}+525 N^{-9}-2227 N^{-10}+\cdots \\
& \mathrm{Wg}^{O(N)}((1) ; 4)=-N^{-5}+N^{-6}-21 N^{-7}+57 N^{-8}-489 N^{-9}+2157 N^{-10}-14077 N^{-11}+\cdots \\
& \mathrm{Wg}^{O(N)}((1) ; 5)=-N^{-6}+N^{-7}-33 N^{-8}+89 N^{-9}-1117 N^{-10}+5237 N^{-11}-45881 N^{-12}+\cdots \\
& \mathrm{Wg}^{O(N)}((1) ; 6)=-N^{-7}+N^{-8}-47 N^{-9}+125 N^{-10}-2123 N^{-11}+10121 N^{-12}-112551 N^{-13}+\cdots
\end{aligned}
$$

$$
\begin{aligned}
& \mathrm{Wg}^{O(N)}((2) ; 3)=2 N^{-5}-6 N^{-6}+34 N^{-7}-126 N^{-8}+546 N^{-9}-2142 N^{-10}+\cdots . \\
& \mathrm{Wg}^{O(N)}((2) ; 4)=2 N^{-6}-6 N^{-7}+64 N^{-8}-300 N^{-9}+2094 N^{-10}-11682 N^{-11}+\cdots \\
& \mathrm{Wg}^{O(N)}((2) ; 5)=2 N^{-7}-6 N^{-8}+98 N^{-9}-490 N^{-10}+4694 N^{-11}-30382 N^{-12}+\cdots . \\
& \mathrm{Wg}^{O(N)}((2) ; 6)=2 N^{-8}-6 N^{-9}+136 N^{-10}-696 N^{-11}+8590 N^{-12}-59850 N^{-13}+\cdots .
\end{aligned}
$$$$
\mathrm{Wg}^{O(N)}\left(\left(1^{2}\right) ; 4\right)=N^{-6}-2 N^{-7}+43 N^{-8}-216 N^{-9}+1737 N^{-10}-10254 N^{-11}+\cdots .
$$$$
\mathrm{Wg}^{O(N)}\left(\left(1^{2}\right) ; 5\right)=N^{-7}-2 N^{-8}+59 N^{-9}-280 N^{-10}+3257 N^{-11}-21934 N^{-12}+\cdots \text {. }
$$$$
\mathrm{Wg}^{O(N)}\left(\left(1^{2}\right) ; 6\right)=N^{-8}-2 N^{-9}+77 N^{-10}-350 N^{-11}+5385 N^{-12}-37498 N^{-13}+\cdots \text {. }
$$

$$
\begin{aligned}
& \mathrm{Wg}^{O(N)}((3) ; 4)=-5 N^{-7}+29 N^{-8}-258 N^{-9}+1590 N^{-10}-10695 N^{-11}+\cdots . \\
& \mathrm{Wg}^{O(N)}((3) ; 5)=-5 N^{-8}+29 N^{-9}-370 N^{-10}+2630 N^{-11}-23815 N^{-12}+\cdots \\
& \mathrm{Wg}^{O(N)}((3) ; 6)=-5 N^{-9}+29 N^{-10}-492 N^{-11}+3738 N^{-12}-42019 N^{-13}+\cdots .
\end{aligned}
$$

$$
\begin{gathered}
\mathrm{Wg}^{O(N)}((2,1) ; 5)=-2 N^{-8}+8 N^{-9}-190 N^{-10}+1460 N^{-11}-15994 N^{-12}+\cdots \\
\mathrm{Wg}^{O(N)}((2,1) ; 6)=-2 N^{-9}+8 N^{-10}-236 N^{-11}+1760 N^{-12}-24254 N^{-13}+\cdots \\
\mathrm{Wg}^{O(N)}\left(\left(1^{3}\right) ; 6\right)=-N^{-9}+3 N^{-10}-120 N^{-11}+742 N^{-12}-13023 N^{-13}+\cdots .
\end{gathered}
$$

$$
\begin{gathered}
\mathrm{Wg}^{O(N)}((4) ; 5)=14 N^{-9}-130 N^{-10}+1640 N^{-11}-14740 N^{-12}+138578 N^{-13}-\cdots \\
\mathrm{Wg}^{O(N)}((4) ; 6)=14 N^{-10}-130 N^{-11}+2060 N^{-12}-20360 N^{-13}+232838 N^{-14}-\cdots \\
\mathrm{Wg}^{O(N)}((3,1) ; 6)=5 N^{-10}-34 N^{-11}+862 N^{-12}-9096 N^{-13}+126523 N^{-14}+\cdots \\
\mathrm{Wg}^{O(N)}((2,2) ; 6)=4 N^{-10}-24 N^{-11}+772 N^{-12}-8436 N^{-13}+121936 N^{-14}+\cdots \\
\mathrm{Wg}^{O(N)}((5) ; 6)=-42 N^{-11}+562 N^{-12}-9426 N^{-13}+114478 N^{-14}-\cdots
\end{gathered}
$$




\subsection{Open questions}

1. (cf. Corollary 3.3.) It is known that the set $\left\{F\left(J_{1}, J_{2}, \ldots, J_{n}\right) \mid F \in \mathbb{S}\right\}$ coincides with the center $\mathcal{Z}_{n}$ of the group algebra $\mathbb{C}\left[S_{n}\right]$. Thus symmetric functions in Jucys-Murphy elements generate $\mathcal{Z}_{n}$. Now the following conjecture is natural.

Conjecture 9.1. The set $\left\{F\left(J_{1}, J_{3}, \ldots, J_{2 n-1}\right) \cdot P_{n} \mid F \in \mathbb{S}\right\}$ coincides with the Hecke algebra $\mathcal{H}_{n}$.

2. (cf. part 4 of Theorem 5.3 and Examples in Subsection 9.1.) We suggest the following conjecture.

Conjecture 9.2. Let $F$ be a symmetric function of degree $k$ and let $\alpha$ be a positive real number. Then, for each partition $\mu \vdash k, \mathcal{A}_{\mu}^{(\alpha)}(F, n)$ is independent of both $\alpha$ and $n$. In particular, for $\lambda, \mu \vdash k, \mathcal{A}_{\mu}^{(\alpha)}\left(m_{\lambda}, n\right)=L_{\mu}^{\lambda}$ and $\mathcal{A}_{\mu}^{(\alpha)}\left(h_{k}, n\right)=\prod_{i=1}^{\ell(\mu)} \operatorname{Cat}_{\mu_{i}}$.

3. (cf. Examples in Subsection 9.1) We suggest the following conjecture.

Conjecture 9.3. Let $F$ be a homogeneous symmetric function of degree $k$ and let $\alpha$ be a positive real number. Then, for each partition $\mu \vdash k-1, \mathcal{A}_{\mu}^{(\alpha)}(F, n)$ is independent of $n$ (but depends on $\alpha$ ).

4. (cf. Theorem 7.3 and Subsection 9.2.) Conjecture9.3 implies that $G_{\mu}^{|\mu|+1}(n)=\mathcal{A}_{\mu}^{(2)}\left(h_{|\mu|+1}, n\right)$ is independent of $n$. Can you evaluate the $G_{\mu}^{|\mu|+1}=G_{\mu}^{|\mu|+1}(n)$ explicitly? From identities in Subsection 9.2, we can obtain

$$
G_{(0)}^{1}=0, \quad G_{(1)}^{2}=1, \quad G_{(2)}^{3}=6, \quad G_{\left(1^{2}\right)}^{3}=2,
$$

and conjecture

$$
\begin{gathered}
G_{(3)}^{4} \stackrel{?}{=} 29, \quad G_{(2,1)}^{4} \stackrel{?}{=} 8, \quad G_{\left(1^{3}\right)}^{4} \stackrel{?}{=} 3, \\
G_{(4)}^{5} \stackrel{?}{=} 130, \quad G_{(3,1)}^{5} \stackrel{?}{=} 34, \quad G_{\left(2^{2}\right)}^{5} \stackrel{\stackrel{?}{=}}{=} 24, \quad G_{(5)}^{6} \stackrel{?}{=} 562 .
\end{gathered}
$$

Recall that the $n$-independent number $G_{\mu}^{|\mu|}=G_{\mu}^{|\mu|}(n)$ is the product of Catalan numbers. How about $G_{\mu}^{|\mu|+1}$ ? We could expect that $G_{\mu}^{|\mu|+1}$ has a good combinatorial interpretation. For one-row partitions, we suggest the following conjecture.

Conjecture 9.4. Let $n, k$ be nonnegative integers such that $n>k$. Then $G_{(k)}^{k+1}(n)$ is independent of $n$ and equal to

$$
4^{k}-\left(\begin{array}{c}
2 k+1 \\
k
\end{array}\right) \text {. }
$$

Equivalently,

$$
\mathrm{Wg}^{O(N)}((k) ; n) \stackrel{?}{=}(-1)^{k} \mathrm{Cat}_{k} N^{-n-k}+(-1)^{k+1}\left(4^{k}-\left(\begin{array}{c}
2 k+1 \\
k
\end{array}\right)\right) N^{-n-k-1}+\mathrm{O}\left(N^{-n-k-2}\right) .
$$


The number $4^{k}-\left(\begin{array}{c}2 k+1 \\ k\end{array}\right)$ is called the area of Catalan paths of length $k$, see [CEF]. Define the set $\mathfrak{E}(k)$ by

$$
\mathfrak{E}(k)=\left\{\begin{array}{l|l}
\left(i_{1}, i_{2}, \ldots, i_{k}\right) \in \mathbb{Z}^{k} & \begin{array}{l}
1 \leq i_{1} \leq i_{2} \leq \cdots \leq i_{k} \leq k \\
i_{p} \geq p(1 \leq p \leq k)
\end{array}
\end{array}\right\}
$$

It is known that

$$
\operatorname{Cat}_{k}=|\mathfrak{E}(k)|, \quad 4^{k}-\left(\begin{array}{c}
2 k+1 \\
4
\end{array}\right)=\sum_{\left(i_{1}, i_{2}, \ldots, i_{k}\right) \in \mathfrak{E}(k)} \sum_{p=1}^{k}\left(2\left(i_{p}-p\right)+1\right) .
$$

\section{Acknowledgements}

The author would like to acknowledge a lot of kind comments with Michel Lassalle and thanks for reviewers' suggestions under a revision.

\section{References}

[BO] A. Borodin and G. Olshanski, Z-measures on partitions and their scaling limits, European J. Combin. 26 (2005), no. 6, 795-834.

[CEF] S.-E. Cheng, S.-P. Eu, and T.-S. Fu, Area of Catalan paths on a checkerboard, European J. Combin. 28 (2007), no. 4, 1331-1344.

[C] B. Collins, Moments and cumulants of polynomial random variables on unitary groups, the Itzykson-Zuber integral, and free probability, Int. Math. Res. Not. 2003, no. 17, 953-982.

[CM] B. Collins and S. Matsumoto, On some properties of orthogonal Weingarten functions, J. Math. Phys. 50, 113516 (2009).

[CS] B. Collins and P. Śniady, Integration with respect to the Haar measure on unitary, orthogonal and symplectic group, Commun. Math. Phys. 264 (2006), 773-795.

[FKMO] S. Fujii, H. Kanno, S. Moriyama, and S. Okada, Instanton calculus and chiral onepoint functions in supersymmetric gauge theories, Adv. Theor. Math. Phys. 12, no.6, (2008), 1401-1428.

[G] A. Garsia, Young's seminormal representation and Murphy elements and content evaluations, Lecture Notes in Algebraic Combinatorics, available at: http://math.ucsd.edu/ garsia/.

[J] A. Jucys, Symmetric polynomials and the center of the symmetric group ring, Reports on Mathematical Physics 5 (1974), 107-112. 
[KOO] S. Kerov, A. Okounkov, and G. Olshanski, The boundary of the Young graph with Jack edge multiplicities, Internat. Math. Res. Notices (1998), no. 4, 173-199.

[LT] A. Lascoux and J.-Y. Thibon, Vertex operators and the class algebras of symmetric groups, Zap. Nauchn. Sem. S.-Peterburg. Otdel. Mat. Inst. Steklov. (POMI) 283 (2001), Teor. Predst. Din. Sist. Komb. i Algoritm. Metody. 6, 156-177, 261; translation in J. Math. Sci. (N. Y.) 121 (2004), no. 3, 2380-2392.

[L1] M. Lassalle, Some combinatorial conjectures for Jack polynomials, Ann. Comb. 2 (1998), no. 1, 61-83.

[L2] M. Lassalle, Jack polynomials and some identities for partitions, Trans. Amer. Math. Soc. 356 (2004), no. 9, 3455-3476 (electronic).

[L3] M. Lassalle, A positivity conjecture for Jack polynomials, Math. Res. Lett. 15 (2008), no. $4,661-681$.

[L3] M. Lassalle, Jack polynomials and free cumulants, Advances in Mathematics 222 (2009), 2227-2269.

[L4] M. Lassalle, available at http://igm.univ-mlv.fr/ ${ }^{\sim}$ lassalle/jack.html.

[Mac] I. G. Macdonald, Symmetric Functions and Hall Polynomials, second ed., Oxford University Press, Oxford, 1995.

[Mat] S. Matsumoto, Jack deformations of Plancherel measures and traceless Gaussian random matrices, Electr. J. Combin. 15 (2008), \#R149.

[MN] S. Matsumoto and J. Novak, Jucys-Murphy elements and unitary matrix integrals, arXiv:0905.1992v2.

[N] J. Novak, Jucys-Murphy elements and Weingarten function, Banach Center Publ. 89 (2010), 231-235.

[OO] A. Okounkov and G. Olshanski, shifted Jack polynomials, binomial formula, and applications, Math. Research Lett. 4 (1997), 69-78

[O] G. Olshanski, Plancherel averages: remarks on a paper by Stanley, Electr. J. Combin. 17 (2010), \#R43.

[Sa] B. E. Sagan, The symmetric group. Representations, combinatorial algorithms, and symmetric functions, second ed., Graduate Texts in Mathematics, 203. SpringerVerlag, New York, 2001.

[St] R. P. Stanley, Some combinatorial properties of hook lengths, contents, and parts of partitions, Ramanujan Journal (in honor of George Andrews for his 70th birthday), to appear. arXiv:0807.0383v3.

[W] D. Weingarten, Asymptotic behavior of group integrals in the limit of infinite rank, J. Mathematical Phys. 19 (1978), no. 5, 999-1001. 
[Z] P. Zinn-Justin, Jucys-Murphy elements and Weingarten matrices, Lett. Math. Phys. 91 (2010), no. 2, 119-127. 